\title{
Les « Monuments français inédits » (1806-1839) de N.-X. Willemin et la découverte des "Antiquités nationales "
}

\section{Françoise Arquié-Bruley}

Volume 10, numéro 2, 1983

URI : https://id.erudit.org/iderudit/1074291ar

DOI : https://doi.org/10.7202/1074291ar

Aller au sommaire du numéro

\section{Éditeur(s)}

UAAC-AAUC (University Art Association of Canada | Association d'art des universités du Canada)

\section{ISSN}

0315-9906 (imprimé)

1918-4778 (numérique)

Découvrir la revue

\section{Citer cet article}

Arquié-Bruley, F. (1983). Les « Monuments français inédits » (1806-1839) de N.-X. Willemin et la découverte des «Antiquités nationales ». RACAR : Revue d'art canadienne / Canadian Art Review, 10(2), 139-156.

https://doi.org/10.7202/1074291ar

\section{Résumé de l'article}

Reference works which marked their day and were improvements over what previously existed are normally taken for granted, eventually forgotten. The in part posthumous work of N.X. Willemin in selecting and publishing the 'National Antiquities' of France, is one of the earlier and more important histories of the arts on a comparative method, treating works front the sixth to seventeenth centuries as documents of the historical evolution of society. The art works discussed were not only in the leading Parisian and provincial museums and libraries, but from outstanding private collections as well, so the Monuments français inédits is the result of a complex series of relations between scholars, editors, collectors and dealers in the transitional period between Revolution and Restoration. Their identification and the characterization of their activities will provide scholars with a more secure foundation for further research in the early Romantic period which is as yet poorly documented.
Tous droits réservés @ UAAC-AAUC (University Art Association of Canada | Association d'art des universités du Canada), 1983
Ce document est protégé par la loi sur le droit d'auteur. L'utilisation des services d'Érudit (y compris la reproduction) est assujettie à sa politique d'utilisation que vous pouvez consulter en ligne.

https://apropos.erudit.org/fr/usagers/politique-dutilisation/ 


\title{
Les «Monuments français inédits » ( I 806-I 839) de N.-X. Willemin et la découverte des "Antiquités nationales»*
}

\author{
FRANÇOISE ARQUIÉ-BRULFY
}

Paris

I.e gon̂t pour l’óponguc médiévale, sa litcíraturce el son art, s'est déceloppé en France plus lardivemont qu'on Angleterre et en Allcmagnc'. Des érudits, des religicux entre autres, avaicnt fait, sous la royautc, un travail inestimable en publiant cle nombreux lexles d'archives. Un cortain public appréciail anssi, arant la Rérolution, le charme des romans commois. Mais on consiclérail l'art du Moven Âge comme barbare, "gothipuc". I.es amateurs qui parfois faisaient place dans leurs cabincts aux objets de ce temps s'cn amusaicm comme de curiosicós. Il faudra attendre les dernières amnócs de la Restamation pour que des collections qui en comprenaient entrent au louvre 2 . Vers 1830 , on admirait, avec les corivains romantiques, les grandes cathédrales er les rumes des châtcaux; les décors néo-gothiques cmahissaient les demeures, et, dans les bals costumés, on ne complait plus roubadours el châtelaincs portant le hemmin.

Pendam le premier quart du siècle, on écrivit très peu sur l'art du Moyen Âge et de la Renaissance, et l'on con fit comnaître encore moins d'images. Les érudits s’intéressaient à l'art antipue, grec, romain el égyptien³. Certains, commc Cambry, se passionnaient pour les origines celtiques de la France'. Si, pendant la Révolution, Millin avait fait rapidement dessiner et graver les monuments qui allaient être détruits ou risquaicnt de l'êtrcin, il avail ensuite consacré à l'Antiquilé la plupart de ses ourrages. Cambry avait décrit et fait reproduire par Naudet des ivoires mécliévaux ${ }^{6}$, alors (juc, premier préfet de l'()isc, il luttait contre le vandalisme.

Répandre dans le public le goût du Moyen Âge et de la Renaissance ${ }^{7}$, donner aux artistes, avant tout aux peinties d'histoire, des modìles d'une exactiucle scientiligue, furent les buts de Nicolas-Xavier Willemin, dessinateur et gravem, antiquaricex, dans ses Monuments fiangais inédits

* Je remereie tom coux qui mont apporte des renscignements pour a maval, en particulier M. Jestat, dircoreur d'études a l'loole des Hautes F́tudes, M. Alcoulfe, comservateur en chef du département des objets d'ant du Muséc du Lomre, Mme (iaborit. M. Lefébure al M. limmes. conscrvalcurs, (1) Virlande-Brandenburg, comservalcur en chef du Musec de (ilum

I Le calaloguc de l'exposition Le "gothiques retromeré areant Viollet-le-l)ur (Hôtcl de Sully, Paris, 31 octobre 1979-17 février 1 g $8(1)$ constitue le meilleur résumć acuucl de la question. Avec bibliographice.

2 Acquisition par k lomve de la collection I) lanand (Antiquité, Renaissance, quclques objets (lu Moyen Agc) en 1825 , co de la collection Révoil (Moyco Age el Renaissance) en 1828

3 Les salumts (fue Bomaparte avait cmmenćs lors de son expédinion en legrple anaient contribué à sa meilleme commatissance

4 Jacques (ambry ( $1749-1807)$ fut l'un des fondatems e le premier president de l'Académie celtipue $(1805)$, alljomrl'hui Socicio nationale des antiquanes de Fance.

5 Aubin-Louis Millin, Antiquilés nationales on Recureil de Momumens pener serair à l'historire générale el particuliere de l'Eimpire fransois, tels que tomboaux, inscriptions, statues, vitranx, fiespues, tirés des abbaves, momasteres, châteanx el antres lienx dezumurs domaines natiomunx (Paris, $1790-1798), 5$ vol. Millin avait tratvaillé dans des conclitions difficiles, devant par excmple solliciter l'autorisation de pouvoir "jetter un coup d'ocil" sur le trésor des (élestins au moment où l'on ferait la levec des scellés (avant de le dipecer et de le fondre) (Arch. Mat., Fir 1036 в). Dibdin, lors de son rovage à Paris en 1,818 devait lui reprocher quclques imperfections (Rev. Thomas lirognall Dibdin, A Biblingraphical Antiquarian and Picturesque Tour in France and (jermany) [Londres, $18211,11,13^{(j-137)}$.

$6 \mathrm{~J}$. Cambry, Inescription du département de l'(Oise (Paris, I)idot l'aîné, an x1, 1803). I a crosse d'Yves de Chartres qui y est gravée est aujourd'hui à Florence au Musée du Bargello; Cambry croyait du Bas-limpire le diptyque du $\mathrm{IX}^{\prime \prime}$ ou $\mathrm{x}^{\prime \prime}$ siècle qui se rrouve manintenant au Muséc dc Cilmuy

7 On ne les séparati jamais à cette époque.

8 Nous employons le mot "antiquaire" dans son sens primitif: colui qui s'applique à l'étude de l'Antiquité. 


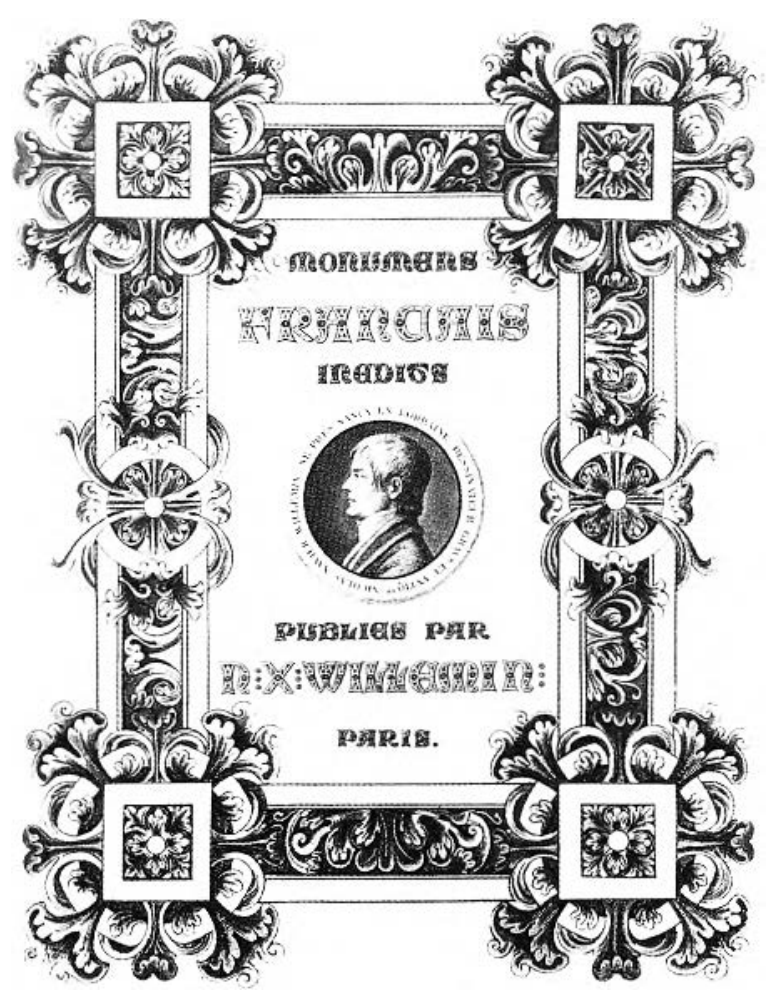

Ficicre: 1. Page de titre de 1839 .

Domic̀e planche exécutéc pour le recucil. I. portrait de llillemin represente l'artiste encore jeunc, arec unc coilfure à la mode dans les demicres amnées du xvm" siècle. Lencadrement somptueux est liré d'un Sacramentaire (Bibl. Rouen, ms. 274), offert par Robert de

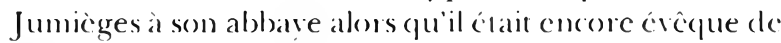
Lonclices (1044-1050). ("est unc nurre capitale de I'Foole ditc de Winchester (Mamuscrits normands, XI-XII' sierles, Muséc des Beaux-Arts, Rouen, fer.mars 1975, texic: de $\mathrm{F}$. $\Lambda$ vril).

9 . Momumemts frangais inédits pour servir à l'histoure des arts depuis Ie I't' siécle jusqu'au commencement du Xvil". C:hoix de costumes cirils et militaires d'armes, armures, inssinuments de musique, menbles de toule especen, "l décorations intérieures al extérieures des maisons, dessinés, grazés el coloriés d'après les originaux par $N . X$. Willemin, auteur du "Choix de costumes civils et militures des proples de l'Antiquitén. Classés chronologiquementel el accompagnés d'un lexte historique "t descriptif par André Pottier, conservateur

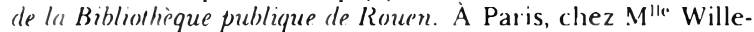
min, rue de Sèrres, n" 19. M.DCcC.xxxix.

10 Françoise: Arquić-Bruley, "Ln précurseur, le comte de Saint-Morys, collectionneur d'Antiquités nationales (1772-1817)". Gazelle des Beaux-Arts, vi/xcvi (octobre 1 (980), 109-118; vi/XCVII (février 1981), 61-77. Jc ne citcrai pas à nouveau cet article pour ne pas alourdir inutilcment les notes. On y trouve en effer de nombreux renseignements sur des objets d'art reproduits par Willcmin et les collectionneurs qui les ont possédés.

11 Ces recherches sont d'autant plus difficiles que l'État civil parisien, brûlé en 1871 , n’a été que très partiellement reconstitué
(Fig. 1)". Mais comme tout précurseur il se heurta à de nombreuses difficuliés. Il s'adressait à un public restreint, dans unc période troubléc; il roulait réussir une ceurre à la fois sarante dans le choix et la reproduction des monuments et luxueuse dans son exécution. Il manqua sourent d'argent; à plusieurs reprises, il crut devoir renoncer. Trente-trois ans s'écoulèrent contre la livaison de la premicre planche er celle de la trois cent deuxième et demière. Commencé en 1806 , l'ourage fut tcrminć en 1839 par André Pottier qui n'avait que sepu ans au débul de sa parution; IVillemin était mort en 1833 , usé par le travail et les soucis.

A notre époque où la photographie permet de répandre l'apparence des oeures dart dans un large public, les chercheurs trouvent dans les $1 / 10-$ muments fransais inédits des renseignements historiques différents de ceux que l'auteur arait l'intention d'apporter. Mais nos contemporains sont sumpris par le désordre des images, le manque d'informations foumies par les sous-titres, que compensent rarement les notices correspondanIcs; enlin ils sc heurtent très vitc au probleme essenticl de la datation des gravures: en 1839 , le texte d'A. Ponticr fut accompagné d'unc table des matières indiquant, pour la reliure, les numéros antribucs aux planches qui, jusque-la, n'en comportaient pas; il sembla par contre inutile de conserver le sourenir de l'ordre de leur paruion. Au cours de mes recherches sur l'histoire des collections d'objets d'art du Moven Âge et de la Renaissance ${ }^{10}$, l'ourrage de Willemin ne m'a ainsi fourni que des renseignements lragmentaires. Pour en extraire un plus grand nombre, j’ai dô me livrer à d'énormes dépouillements tant d'archives que d'imprimés et reconstituer la vie d'hommes dont je ne connaissais que le nom patronymiçue, souvent mal orthographić"'. Mes questions n'ont pas toutes trouré de réponse; mais il me paraît utile de publier le résultat de mon travail, même incomplet, en le dédiant aux historiens d'arl qui s'en scrviront et qui, je l'espère, le pousscront plus loin.

Rejetant à la fin de l'article des listes de dates, je ferai d'abord connaître ce que j'ai entrevu de l'histoire de ce recueil pour mieux le faire comprendre et permettre de l'utiliser. Cette histoire est étroitement liée, à travers les changements de régimes, à celle de l'auteur et à celles d'amatcurs, d'ćrudits, d'artistes qui voulurent la réalisation de cette ouvre et apportèrent leur secours à une entreprise en laquelle ils croyaient. 


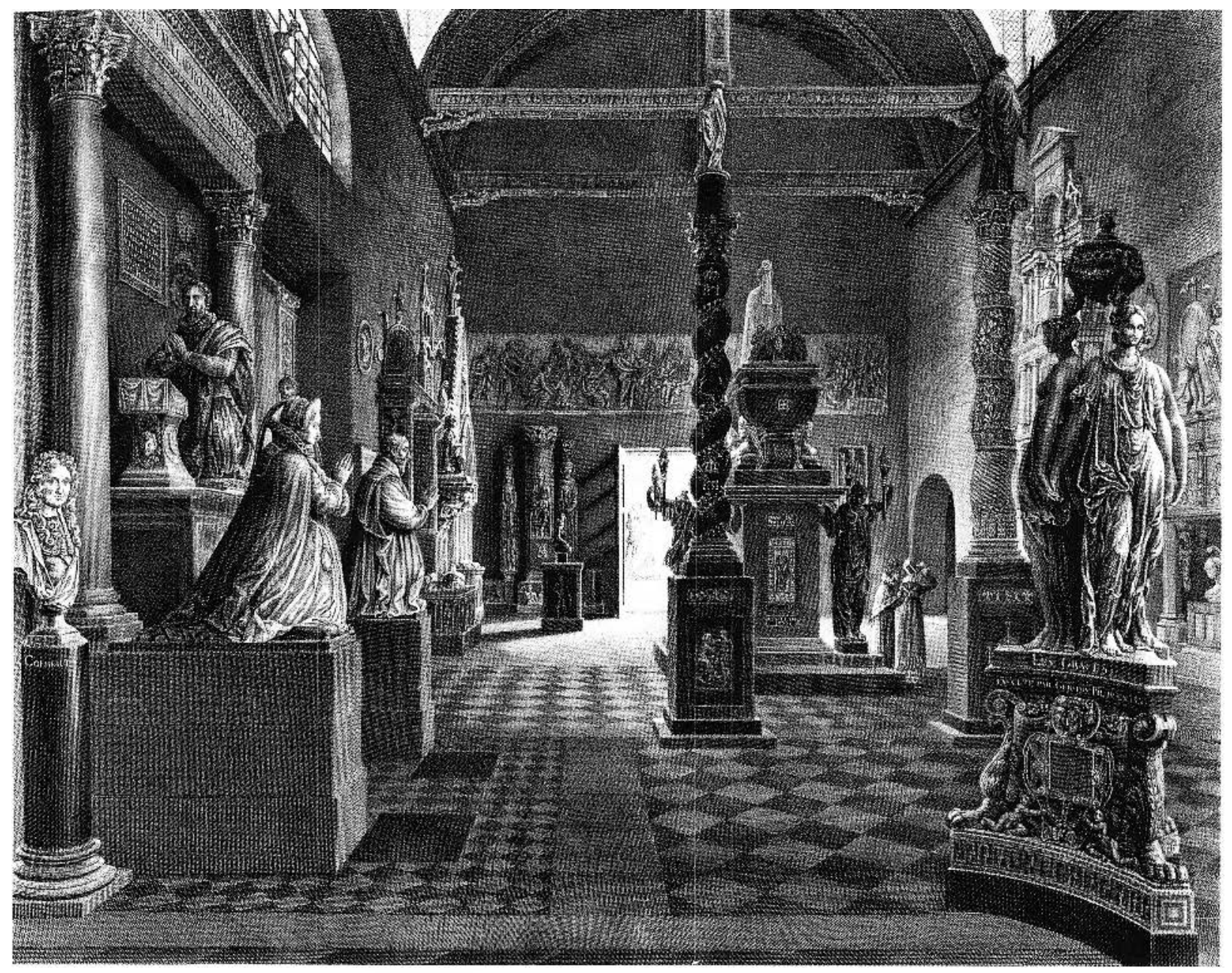

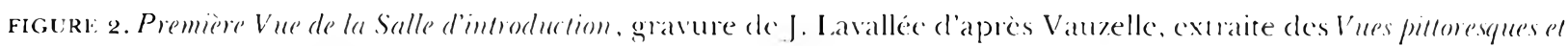

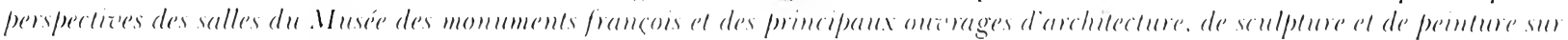

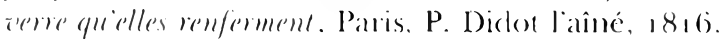

Nicolas-Xarier Willemin naquit près de Nancy vers $17^{6} 7^{12}$. Ses parcons. Joseph-Xavier Willemin et Barbe Petamant dit Vilai, vintent peu après s'installer à Paris. Lenfant apprit la gravure che\% un roisin, puis il se porfectionna en dessin avec, pour man̂tres, laillasson et Lagrenée le jeune. Désircux de gagner sa vic, il se chargea d'ceurres fort variées: portraits, fac-similés de Bartolozzi, Cahicrs d'ornements gress et romains..

Le $1^{\text {cr }}$ germinal an $\mathbf{m}$ ( 21 mars 1795 ), Willemin. "grarcur en taille-doucc et dessinateur à l'administration des assignats", épousa la jeunc veure d'un sculpteur des Mcnus Plaisirs, VicroireÉticmncue Bihc1'3. dont il cur un fils. AugustePhiclias't.

Willemin étail un travailleur acharné. Il décida, dans les dernic̀res années du siècle, de se lancer dans une publication gui lui assura une grande notoricić: Choix de costumes cirils et militaries des
12. Nicolas-Xavier llillemin ast né près de Xancy peut-être

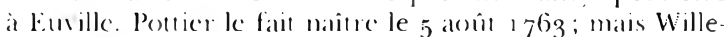
min declare aroir 28 ans (âge sans doutc approximatur), k

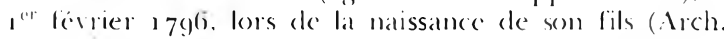
Parris. Fital civil reconstiunc)

13 I cesomcles de la jeune fomme étaient, arant la Rivolution, "bourgeois de Paris": l'un chail cmployé des formes gé

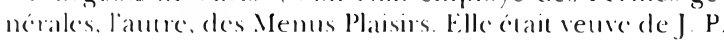
Pumman, liégeois doriginc, fils d'un matbrier (Archonat. Minutice cemmal|m.c.]. xxxu, 60. 24 livitier 1792 , contrat de matriage Putman-Bihet: $x \times 11,114,15$ frimaire an 11 [5 dicombre 1794 ], inventaire apress déces de J. P' Puman

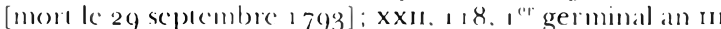
[21 mats 1795 |. martage Willemin-Puman]. . I'me Willemin avait un fils de som premicr mariage: Antome-Swamme. Il mourta à Séville (lispagnc), rompellc d'un régiment de grenadiers, k 7 octobre 1811 (M.c... cxxi, 9 mats 1813 , notoricié du decoss d't - S. Pumant.)

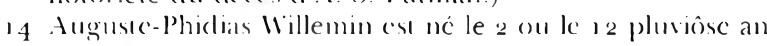

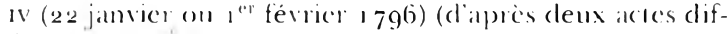
férents de l'stat civil reconstitué). Il était en vic all moment de la mort de sa mere en 1813 . Je naa pas trouve ware de son cléces qui se silue forcement arant le 26 novembre 1831. date à laquelle Willemin adopta unc jeune fille. 
peuples de l'Anliquitél.j. Ces 180 planches, tiréces à $45^{\circ}$ excmplaires et vendues par livraisons de 1798 à 1806 , se recommandaient par lcur sérieux; vêtements, instruments de musique, ustensiles élaicn copiés dans les meilleurs ourrages des dernières décennies ou dessinćs, plus rarement, dans des cabinets d'amateurs, el devaient servir de modèles aux artistes soucieux de vérité historique $^{16}$. Le texte signé de Willcmin était en réalité de l'abbé de Tersan'i, antiquaire connu, mais aussi discret qu'érudit. L'œuvre eut un grand

Ij Choix de costumes cionls et miluarires des peuples de l'Auliqunté, lenurs instruments de musique, leurs menbless, al les décoralions intérienues de leurs maisoms, d'après les. Momumens antiques, auee un texte lire des anciens auteurs (Paris, 1 798-1802). Les clernières livraisons parurcut en réalité cn 1806 .

16 Cincuantc ans auparavant, le come de Caylus avait fait graver les objets de son cabinet pour fournir des molèles exacts atux artistes: Recueil d'antiquités égyptiennes, émusques, grecpues "l romaines (Paris, $175^{2-1767)}$

17 Charles-Philippe Campion de Tersan (Marseille, 8 aôt 1737 - Paris, 11 mai 1819 ). Lui-même avait fait graver des antiquités de sa collection, mais ne les avait pas publićs. Après sa mort Grivaud de la Vincelle s'en servit pour illustrer un de ses propres ourrages, Arts et métiers des anciens peuples (Paris, 1819 ).

18 A Frande-Brandenburg, "Le Musćc des Monuments fransais co les origines (lu Musée de (:luny", Das Kunst und Kulturgeschchtliche Museum im 19. Jahrhundert (Munich, 1977), 49-58. B. Foucart, "La fortune critique d'Alexandre I.enoir et du premier Musée des Monuments liançais", L'Information d'historere de l'arl (1 g(fog), 223-232. Willemin élaut domicilić au Musée des Monmments français en 1798 (parge de titre du Choix de coshumes cirvils ol militures...) et 1800 (I.inrel du Salon de I 800$)$. I c: Musée était situc dans les batiments de l'ancien couvent des Potits-Augustins, a l'emplacoment de l'Focole des Bcaux-Arts. Willemin lui avait lait don d'unc Ammonciation d'albâtre (Alexandre I.enoir, Description historique el chromologigue des momuments de sculpture réunis au Musée des Monumenls frangsais. . [Paris, an vill, 1799 ], 159, " $^{\circ} 432$ ).

19 Les gisants, par excmple, étaient somvent dressés contre les murs.

20) Ainsi la chapelle sépulcrale d'Hélö̈sc ct d'Abélard.

21 La se trouvaient la plupart des tombeaux.

22 André Rostand, "La documentation iconographique des Monumens de la Monarstie françosse de Bcrnard de Montfaucon " dans Bullelin de la Société de l'histoire de l'art francais (1932). l'auteur insiste (p. 148) sur l'“impuissance des dessinateurs de ce temps-là à rendre le caractère des œuvres d'arı du moycn-âge". Il ajourc (p. 149) que "l'extrême délicatesse de cet arl les rebute: c'est le terme que l'on a vu employé à plus l'une reprise".

23 Dubut, (iau, Le Bas, Malpièce, Van Clemputte.

24 Paul l urmcau. né à Paris. ! mouruc le: 3 décembre 1849 à l'âge de 77 ans (État civil reconstitué). ("était. comme nous l'apprend son inventaire après décès, In amateur d'objets d'arı du Moyen Âge ct de la Renaissance, de boiscries sculprécs ct de musigue ancienne (M.c., I.XIV, 755, 20) décembre 1849).

25 François Debret (Paris, 27 juin 1777 - Saint-Clond. 19 lérice 1850 ). Il (lessmait aussi (des objess appartenant à des clicnts collectionncurs

26 Etiennc-François Imbard (Annonay, 1780 - Versailles, 13 aviil 1830). Catalogue des tableaux, dessins, estampes... prozenamu du cabinet de fen ، M'F.F. Imbard, par Duchesnc âné, les $28 \ldots$ novembre 1831 . Hôtel Bullion. Arec noticc nécrologicue. rayonnement; parmi les souscripteurs, on trouve les noms du ragédien Talma, des peintres David, Girodet, Guérin, Prud'hon, Révoil, de l'orfèvre Auguste, du ciseleur Thomire, enfin des grands collectionneurs, Denon, Van Hoorn et Iufourny.

Ia réussite de cet ouvrage poussa Willemin à en commencer un autre, plus ambitieux, et sur un sujet d'actualité: les Antiquités nationales.

Willemin habitait à ce moment l'endroit même où artistes et érudits se retrouraient dans leur amour du Moyen Âge, le Musée des Monuments français ${ }^{18}$. D'abord dépôt d'oxuvres d'art pendant la Révolution, il devint, grâce à Alexandre Lenoir, un musée de sculptures, vitraux, objets d'art (Fig. 2). Regroupés de façon arbitrairc, modifiés au besoin, exposés de manicre illogique ${ }^{19}$, formant parfois d'étranges monuments composites ${ }^{20}$, ils constituèrent pourtant des ensembles qui impressionnèrent les contemporains, et leur firent comprendre qu'avant l'époque classique existaient dans leur pays d'autres formes d'art.

W'illemin qui, pour les recueils précédents, dessinait et gravait toutes les planches, s'adjoignit cclle fois des collaborateurs. Il les choisit tout naturellement parmi les nombreux artistes qui fréquentaient assidûment les salles des PetitsAugustins et le jardin Elysćc ${ }^{21}$. Formés à l'origine par la copie d'antiques, ils s'étaient imprégnés d'un cesprit nouveau et n'étaient pas rebutés par les formes "cćlicates" du "gothique" comme, près d'un siècle plus tôt, lcurs prédécesseurs incapables de dessiner pour Montfaucon ${ }^{22}$. Willemin, soucieux d'exactiude archéologique, s'adressa surtout à des archicctes. L'aide de certains fut épisodique ${ }^{2.3}$; d'autres tinrent une grande place. Paul Turmea $u^{24}$. dans les premiers temps surtout. fournit trente-deux modìles au graveur. Nous trouvons quinze fois le nom de François Debret ${ }^{25}$. Il rapportail de ses voyages en Brotagne, en Italic, en Allemagne, des relevés de chapileaux, de meubles. Celui qui esi pour nous le restaurateur malheurcux de Saint-1)enis a joué un rôle important dans le retour au "gothique". Imbard ${ }^{26}$, jeune peintre sans argent devenu architcete par nécessité, secondait Alavoine, alors chargé cles laavaux du Musée des Monuments français. Il fil quelques dessins pour $\Lambda$ lexandre I.cnoir et Willomin, obtint d'installer son atelier dans les vastes bâtiments conventuels, publia des gravures des tombeaux de Iouis xir el de François $\mathrm{I}^{\mathrm{Tr}}$

Les dessins d'architectes peuvent être admirés pour lcur exactitude, leur neteté, mais ils rebutell nos contemporains par lcur froidcur. Debret domnc une apparence presque néo-classique à un 

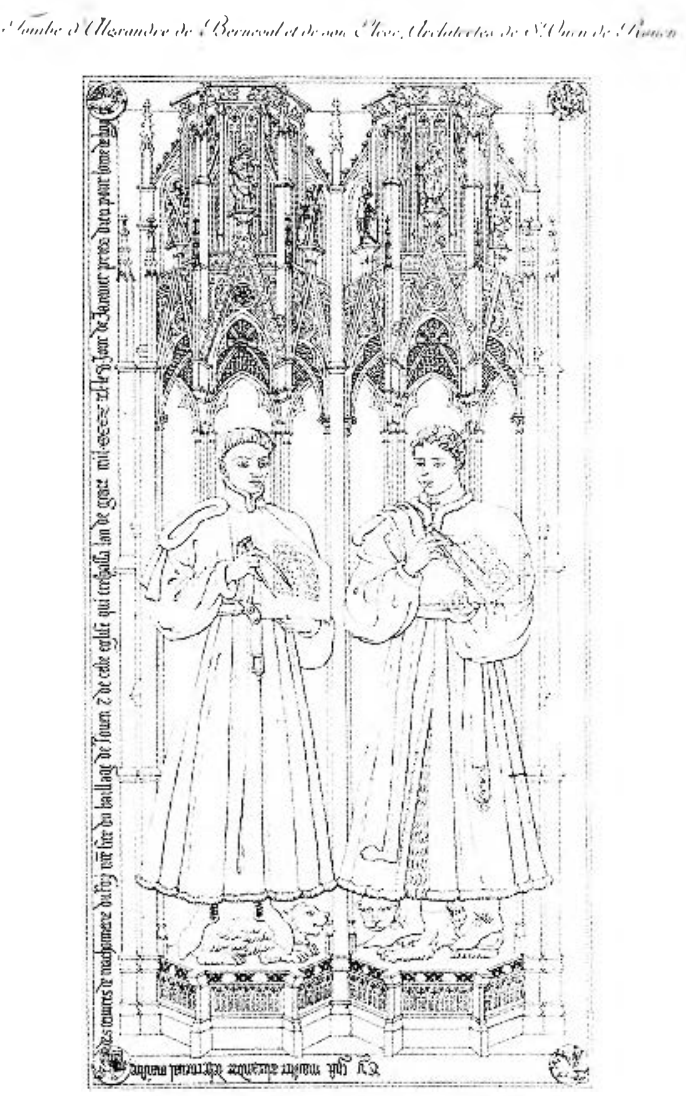

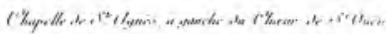

FIGURE 3. Pl. 159 des Momuments fransais inedils. Tombe d'Alexandic de Berneval el de son élève, architerıs de Saint-Ouen de Rouen. Chapelle de sainte Agnes, a gauche du chøur de Saint-()uen. A. Garncrer. Langlois sculp'

Willemin a voulu opposcr la représentation de la lombo de Berneval par Auguste Gameres, à celle, fautive, du dessinateur de (iaigniores (pl. 160).

chandelicr vénitien² ; plus tard, c'est du "gothique troubadour" que se rapproche une copic d'enluminure ${ }^{2 x}$.

Willemin fit aussi appel à deux membres de la famille Carncrev'²9, le père, François-Jean, qui avait accompagné Millin dans ses voyages pour les Antiquités nationales, cr Auguste, un de ses fils (Fig. 3-4).

I.es premières livraisons comprenaicmt déjà des gravures d'aprìs des dessins "du collaboratcur le plus assidu et le plus habile "30 de Willemin. Hyacinthe Langlois, représentant des sculptures, un vitrail, recueillis par Alexandre Lenoir, des staucs de Saint-Germain-l'Auxerrois et des enluminurcs. Willemin appréciait "cettc précision ferme dans les contours... ce modelé soutenu dans les formes... ce scrupulcux rendu de tous les détails,
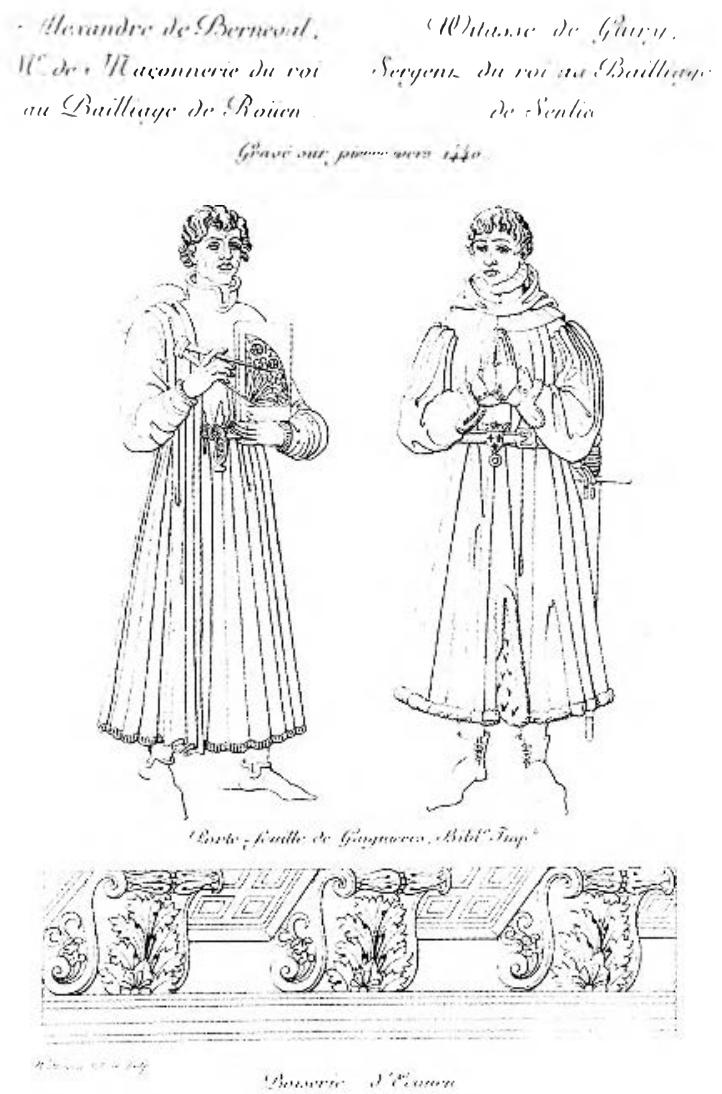

FICURE 1. Pl. 160 des Monuments français inédils. Alexandre de Berneval. I" de maçonnerie du roi an bailliage de Roucn. Witasse de Guiry, sergent du roi au bailliage de Senlis, grave sur pierre. vers 1480 . Willemin del. et sculp).

copiés dans le recucil de (iangniòres.

27 Planche 282

28 Planche 214

29 Sept planches sont signées "Garncrey", une "T F. Garncrev" (pour J.F.), et neuf, "Augustc Garncres". liançoisJean (Paris. 28 déccmbre 1755 - Aureuil. 11 jum 1837) el son fils Auguste écrivaient généralement leur nom "Gannerce" (...., t.xxir. 750. 31 mars 1840 . Votorićté rectifi(ative du nom de (immerai).

3o Citations de Pottier. I anglois fil 35 dessins es 8 gratures pour les Monmments francais imédits. Eustache-H lacindhe Langlois (Pom-de-l'Arche. 3 aoui 1777 - Roucor, 29 scp)lembre 1837). Catalogue de lexposition E. II. Langlois (Bibliothecpue municipale, Rouen. 1977). arec bibliegraphic :

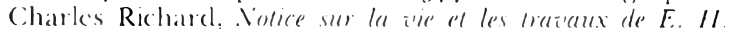
Langlois dlu Pont-de-l'Arche (Rouen, 1838): notice d'A. Por-

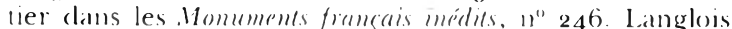
arair été éleve de larvid. Malgré son talent, sa famille comnul une cxtrême misère due à son manque de sens pratiçuc. mais plus encorce à l'alcoolisme de sa lemme. jusquen 1828 . date a laquelle la duchesse de Bom lui fit obtenir une place de professcur de dessin a l'École municipate de Roucn. Deux de ses enfamts. Espórance or Polsclès, collaborerent arec lui. puis travaillèrent pour la Mamufacture de Sevres. 
qui constituait le principal caractère de son talent". La pauvreté de Langlois l'obligea à quitter Paris en 1806 , d'abord pour Pont-de-l'Arche, son pays natal, puis Rouen ${ }^{31}$. De Normandie il continua à envoyer des dessins; Willemin, lorsqu'il s'irritait du manque d'exactitude de ses aides, faisail appel à lui.

Mais le principal dessinateur des Monuments français inédits était N.-X. Willemin lui-même, que les lecteurs avaient l'habitude de voir penché sur

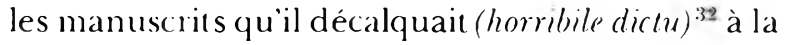
Bibliothèque (qu'elle fut impériale ou royale), ce qui ne l'empêcha pas de reproduire des objets de collection ou, plus rarement, des sculptures ${ }^{3: 3}$. Willemin fut aussi le principal graveur des planches pendant les premières années. Les estampes étaicnt de trois sortes, assure une réclame, "ombrécs, coloriées ou au trait". Flles sont plus satisfaisantes que celles de Ransonnelte exécutées pour Millin, ou celles de Naudet pour Cambry; quant à celles de Laurent Guyot, destinées à Alexandre Lenoir, elles sont loin de les égaler ${ }^{34}$.

Willemin était soutcmu intellectucllement par son entourage: amateurs d'arı médićval, membres de l'Académie celtique, devenue Société impériale des antiquaires de France ${ }^{35}$, qui se réunissait au Musée des Monuments français. Leur

31 Il dessina "t grava de nombreux monuments de Normandic. Il était célèbrc en Grande-Brctagne, or les insulaires passant par Rouen (Dibdin, Walter Scott...) venaient lui rendre visitc.

32 Diblin, II, 492: "Indeed I hardly visited the Public Library without finding $\mathrm{M}$. Willemin busicd, with his pencil and tracing paper, with some ancient illuminated ms."

33 A reouch.

34 Cess illustrations, oxuvres peut-être d’élèves de Guyot, ont été exécutées rapidement ct n'ont généralement pas été inversées pour la gravure.

35 Willemin cntrera en 1823 à la Société royale des antiquaires de lirance.

$3^{6}$ Rostand, 110 . Il renoncera aussi à faire copper l'Histoire des rovs de France de Dutillet.

37 Voir Appendice III.

38 Recueil des costumes franguis depuis Clonis jusqu'à Lomis $x / 1$ imclusierement, dédic à s.m. I'Impératrice el reine, récligé ct publié par L. Rathier, dessiné par F. Bcaunier.

39 Lauteur fait remarquer sur les statucs reproduites dans la première lirraison. "des marques de dégradation que nous ne nous permettrons pas de faire disparaitre".

40 IDibdin, II. 193: "Six splendid cabinets filled w'th curiositics."

41 Ainsi, en mars 1809 , Lenoir écrit à la suite de comptes au sujer de vitraux achetés à Ficoucn à la vcuve Pétréc: "M. Dufourny a Perre ont acheté à la même femme plusieurs autres panneaux semblables. M. Willemin de même." (Arch. Iouvre, z. 62). compétence, lcur esprit critique, rendaient Willemin de plus en plus exigcant au sujet de ses sources. Il renonça ainsi rapidement, après comparaison avec les monuments eux-mêmes, à utiliser les dessins exćculés pour Gaignières de 1670 à 1715 . Les inexactitudes étaient nombrcuscs, surtout dans ceux destinés à une série "costumes" où les gisants étaient redressés et animés ${ }^{36}$. Il s’attacha donc à reproduire des monuments originaux.

Millin, conservateur du Cabinel des médailles, annonçait dans le Magasin encyclopédique, revue qu'il diffusait dans toute l'Furope, chaque livraison de l'ouvrage de Willemin en énumérant les sujets des planches. Mais les souscripteurs étaient trop peu nombreux. À plusieurs reprises Willemin dut ralentir la parution, ou même la suspendre pendant plusieurs années. Au lieu d'une livraison tous les deux mois, il y en eut six en cinq $\mathrm{anss}^{37}$.

la publication d'un ouvrage concurrent l'atteignit crucllement. Le Recueil de costumes français de Beaunier et Rathier ${ }^{38}$, paraissant à partir de 1809 , était lui aussi destiné à fournir des modèles aux aristes. Il étail moins luxueux: pas d'enluminures, et les gravures étaient exécutécs au trait, rarement ombrées; mais des notices explicatives accompagnaient chaque livraison, ce qui manquait cruellement aux Monuments français inédits; les portraits des personnages historiques se succédaient dans l'ordre chronologique; les gravures sc voulaient plus exactes que celles déjà comnucs"s. Fn réalité, Rathier commettait de grossières erreurs de dates; il laisait recopier Montfaucon dans ce quil a de plus contestable; plus grave, il attribuait au haut Moyen Âge des objets contemporains, telle la "couronne de Charlemagne " exécurée à l'occasion du sacre de Napoléon. On n'y constatait pas la recherche de variété de W'illemin; mais, chaque mois, les souscripteurs avaient la satisfaction de recevoir la suite d'un ouvrage trois fois moins cher et livré régulièrement. Cela dura trois ans; puis la publication s'interrompit à plusicurs reprises pour cesser définitivement en avril 1813 .

Willemin avait connu une certaine aisance et rassemblé une importante collection ${ }^{40}$, acquise à très faible prix. À la suite des bouleversements entrainés par la Révolution, d'innombrables objets apparaissaient dans les salles des ventes et chez les marchands. Les premiers amateurs d'antiquités nationales, même Langlois en Normandie, pouvaient acheter des chef's-d'œuvre. Alexandre Lenoir montrait l'exemple ${ }^{41}$. Mais de graves soucis d'argent obligèrent N.-X. Willemin à disperser son cabinet au profil, entre autres, de Révoil, 
Saint-Morys et Saurageot ${ }^{+2}$. Par la suite, il acheta, revendit, servit d’intermédiaire, mais n'eut plus de collection persommellet.3.

Il diversifia ses travaux, revenant à l'Antiquité qui se vendait mieux; Lenoir, conservateur des objets d'art de la Malmaison, obtint pour lui avec la protection de l'impératrice Joséphine, la commande d'estampes des peintures, vases et bronzes antiques de celle-ci. la première livraison parut en $1810^{\prime \prime}$. L'artiste dut alors se faire aider pour les Monuments français inédits par plusieurs autres

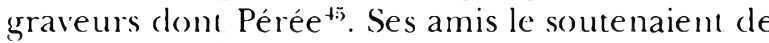
leur mieux en recommandant son ouvragetti. L'espoir revenait. Les lecteurs araient reçu scpt cnrois de planches de 1810 à 1812 , lorsque Willemin perdit sa femme, le 21 juin 1813 . $\Lambda$ sa douleur se joignaient les soucis causés par ses det$t^{4} \mathrm{~s}^{47}$. El dans les années troublées que furent 1814 et 1815 , qui put se soucier d'une œurre tclle que la sicmne?

Mais dès le calme revenu ses amis se manifestìrent. Le comte de Saint-Morys qui avait retrouvé son titre et, espérait-il, une partic de sa fortune engagea Willemin à lancer sur le même sujet unc suite lithographiée, donc moins onéreusc. Sa mort tragique en 1817 mit fin à celte entreprise; mais il avait eu le temps d'introduire le graveur dans le milieu de la cour. Le vicomte de Senonnes ${ }^{\text {th }}$, secrélaire général des Musées, le comte de Mailly, pair de France el aide de camp du duc de Berry, obtinrent de ce "prince ami des arts" qu'il acceptât la dédicace des Monuments français inédits. La protection de la famille royale lui demeura acquise après l'assassinat du duc.

Willemin reprit sa tâche avec un nouveau courage. Il n'était pas riche, mais il était apprécić, cutouré, admiré; des étrangers de passage à Paris lui rendaient visite; des artistes ćtudiaient son courret". Ln 1824, il obtint même une méclaille (l'or au Salon ${ }^{50}$. Il se fit aider par de jeunes femmes, chargées d'enluminer les estampes. les planches furent, pour la plupart, gravées désormais par Amédée Pérée, neveu de Jean-louis Pérée ${ }^{51}$.

Willemin fit plusicurs voyages en province à la recherche de sujets intéressants et variés. Il visita Scns, Troyes ${ }^{52}$, Dijon, Amiens, et, de nouveau, Beauvais, y rencontrant érudils el collectionneurs. À Strasbourg, il exécuta les illustrations de l'ouvrage d'Engelhardt $(1818)$ représentant des dessins de l'Hortus deliciarum de l'abbesse Herrade de Landsbergi3.

Il décida cle donner de l'extension à son ouvrage; les cent cinquante planches prévues au début devinrent deux cents ${ }^{54}$, pour finir par être trois cents. Des vignettes devaient être intercalées dans le texte qu'il commença à publier en $1825 \mathrm{et}$ $1827^{55}$. Un problème se posait à lui : il ne trouvait guère en France, à part les enluminures carolingiennes, d'œuvres du haut Moyen Âge; il fut

42 Le nom de Rćvoil ne figure que comme dessinatcur d'un pilastre du cloîre de Saint-Sauveur à Aix-en-Provence (pl. $3^{8)}$ ). Pourtamt certains objets indiqués comme fatisant partie de la collection de l'autcur sont entrés au Ioure avec la collection de Pierre Révoil (Lyon, 12 juin $1776-$ Paris, 20 mars 1842), peintre d'histoire: cas(fue, pl. 255 (Call. Courajod, 3); conteau d'ivoire, pl. 284 (Courajod, 159), actuellement au Musce de Cluny (C:1. 22.245); les tissus des planches 78 et 113 (Courajod, 386 ct 387 ) soml aussi au Musce de Cluns. I e dessin d'unc jeunc fille du $\mathrm{Xv}^{*}$ siècle. pl. 165 , qui ne porte pas de nom de collectionneur, est entré cn même temps au Lourre (Courajod, 281). L. Comarjod, La Collection Rézoil du Musée du l.onire (Caen, 1886 ) (cxtrait du Bulletin Monumental). La poire à poudre, pl. 284 , avec le portraic de Gabriclle d'Esnées(⿳), est entróc au Lourre (Molinier, 186) avec son pendant représentant Henri Iv. après passage clans les collecrions Sainl-.Mory's et Roger.

43 L.es chandeliers. pl. 282 et 283 , onl été dessinés cn 1817 par loebret alors quils étaient dans la collection SaintMorys et au moment de la mont de celui-ci. Ont-ils apparromu au "Cabiner de l'auteur" aupararant. ou au contraire été acquis à la vonte Saint-Morys (26 janvier 1818) pour ctrc aussitôt revendus (Willemin nc possédait pas un seul objet d'art lors de la visite de I)ibdin, le 8 juillet 1818 )? On nc sail ce qu'est devenue une de ces paires de chandeliers (sur laquelle $M$. Jestaz a recommu les armes des (iiustinian (le Venise). Lautre est entréc au I ouvre avec la collection Sallvageot (OA 719 et $7328-7329$ )

44 Fn 1811 , il commonça aussi à fairc parâtre un Parallèle des plus anciemnes peimlures el sculphures antiques.... qui n'out que quelques livaisons.

45 Jacques-Louis P’éréc, né dans l'()isc en 1769, mort à Paris le 8 avril 1832 (lian civil reconstimé).

46 M. de Saint-Morrs écrivit un article élogieux sur l'ourrage de Willemin dans le Journal de lo ()ise du 9 juin $1812 \mathrm{cl}$ dans le Joumal des arts, des sciences et de la littérature du 1 j juillet $1812,56-57$. Il présenta aussi l'artiste au mare de Beauvais ct aux collectionneurs de la région.

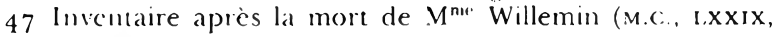
$4(j(j, 2$ juillet 1813$)$. Willemin avait des dettes convers ses amis el son foumisseur de papier.

$4^{8}$ Plusicurs des persomnages cités sont des collectionneurs anxulucls est consacré l'Appendice I.

49 Dans un carnet d'études de Delacroix figurent des copies de détails de plusicurs planches des Mormumemes /rançais imédits. M. Swar\%onski a communiqué ce renscignement à

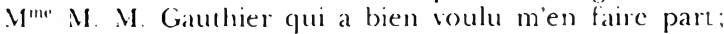
quils con soient tous deux remerciés.

50) Willemin ne reşut sa médaille d'or qu'en 1825 , la mort de l.ouis xvıl ayant retardé la remise les récompenses.

51 Plusieurs gravures sont signées Pérée, oncle cl neveu Jacques-Louis-Amédés Pérée, né à Paris le 26 juilled 1797 (Fial civil reconstituc), fils de Jcan-louis Pérć (Feuquières, 3o décembre 1771 - Paris, 30 avril 1803), graveur en taille-douce. Amédéc Pérée a été oublié par les dictionnaires d'artistes; il avait pourtant gravé cent trente-trois planches des Monmments français inédits.

$5^{2}$ Ammales françaises de's arts, des sciencies or des lellre's (1821-22), $294-302$.

j3 I.e mamuscrit a été (létruit en 1870

54 Prospectus de 1821

55 Il reste la vigneite de la rare page de citre de 1825 . La planche 111 ou figurent des émaux du conte de Mailly, porte, sur certains tirages, les indications: Vignctte $w^{*}$ palge 26 . 
amené à copier quelques illustrations de livres étrangers, faisant mentir ainsi le linc de sa publiation: les monuments reproduits n’étaicnt plus

$5^{(j)}$ Planches 19 à 23 . Délméation exacte des ormements impéminux du. Samb-Fimpire romain allemand. gardes dams la rille libre al impériale de Nuremberg, dessinćs et graves aux dépens de

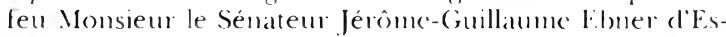
chenbach. par Jean-Acham Delsembach. Aver les samtes

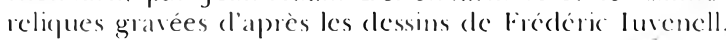
fun douze planches gravécs are une descriplion. A Nuremberg, chez Adam (ionliel) Schucider, 1790 .

j7 Pl. 3. empereurs de porphrre; pl. 5, sioges ćpiscopanx;

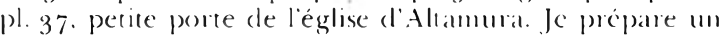
article sur ce fonds iconographicpuc.

58 A. Pottier. Arant-propos.

jo) Willemin visait matrialement are (inevieve-Joséphine Bron-Bozon, qui se faisail appeder " Mme Milleminn. Elle

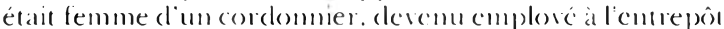
des vins. Nicolas Marotte qui mommo ilhojpital de la Pitié le 19 avil 1838 (Arch. Paris. De ${ }^{*} 10,13$ ). Helle épousa par la suite un cortatin Frangois Ratel. ex momul veme une nou

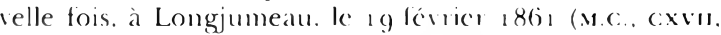
1312. 31 mai 1866). I.es époux Marnte arachl eu au moins deux enfants: Marguerite-(iabriclle, nee le 28 aril 1810 (Etat covil reconstimé), qui deviendra la fille adoptive de Willemin. el Nicolas, né én mai 1819 e abandommé anssitot aux Enfants-troucs. Iorspuil mommut, le g dé. cembre 1848 . sa mère el sa sceur étaticht ses seules hériliè-

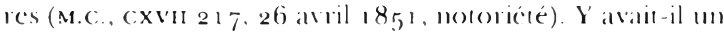
ature ficie. cleve par Willemin? Plusicurs des dernieres planches des.Mommmemts fiancas médie portem la sigualture d'Alphonse Marote Fil dans un prospecous pour lop Ars

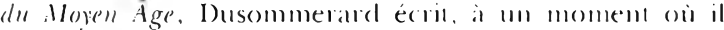
pensaic faire une place importante an burin dams som ouvage: "M. Willemin (Alphonse), collaboralcur de som père dans le bel ourrage des ilommmemh módils mons a déjà gravé seize planches d’un beau lraval qu'il ćlondra a lous les objects dome la reproduction exige mon lain pur el pre cis." On peul penser (fuc at Alphomse Willomin est Alphomse Viarote et quil momut jemme.

(o) Flle a signé quatre fois comme dessmallewr; quatre glatu

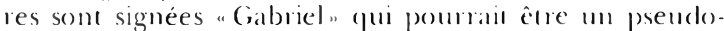
nume transparent. (abbrielle arait pu aussi apprendre le dessin avec Barbier-llalbomme. peimme d'histone, an service duquel elle était depuis a 828 . II limil par l'cpouscr con 1837.

61 André-Ariodant Porticl (Paris. 2 nowombre 1799 Rouen. 26 avil 186-). Roucmans (il rencomman $\mathrm{r}$. II langlois (ans sa ville). il vint a Paris pour ses éludes de méclecine: il s fir la commassance de lomont laalor. Dodier. Willemin; puis il soricnta vers les lentres. II devint conservateur de la Biblionhèque de Roucn on 1833, el, quelques annces plus tand, conservatcur du Huséc d'antiquirés. Il écrivit surtoul sur l’arle el cul particulier la failence. Sa bibliographic a exe publice parr labbe (colas et

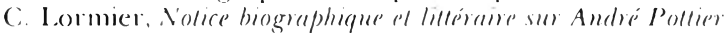
(Rouen, 1868).

(i2 Planches 77 et 297

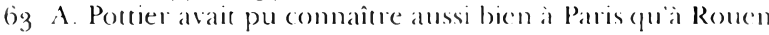
M. de Monville qui possédait des usines pries de cente dernière ville. Pottier démichait che\% les antiquaires romemans des objets destinés à la collection Samvageot (voir Appendice 1$)$. Il est normal de ne pas mourer (lams les Momments firmsais inédits d'oeures appartenam à Alexandre Dusommerard qui, à cete épopuc, se prépalrait à publice les.s .41:s du . Hoje'n Äge

64 Ammonçant la parution d'un texte, vers 1825 , il signale quiun homme de letres commu(ڤ), M. F. de Samm-l.éger. reverra son rexte. André Pottier écrit que Willemin étail un "antiquaire pratique d'un diagnostic lies sion. mais peu habitué à formuler ses idlées" (Avamt-propos). tous "français" ni "inédits". Il fut tout particulièrement séduit par le magnifique recueil d'estampes peintes à la main et représentant les ornements du sacre des cmpereurs romainsgermaniques. Il leur consacra cing planchesiti. Pour l'Italie, il puisa dans le fonds de dessins exécutés sous la direction de Millin, acheté après la mort du conservateur des Méclailles par le Cabinet des Estampes. ${ }^{57}$.

Les souscripteurs reçurent huit livaisons de 1825 à 1827 . puis le rylhme se ralculit. I es forces de Willemin cléclinaicnt, sa vue baissait de façon inquiétante. Il publiait pourtant de nourclles planches lorsquen juillet 1830 Charles $x$ ful renrersé. Lne fois de plus, les protecteurs de l'artiste perdaient toute influence el quittaicnt la capilale, le laissant arec son œurre inacheréc el clans la misère. "Ses facultés intelleculuelles restèrent scnsiblement dérangées ${ }^{\bar{j}}$."

Deux personnes vinrent à son sccours. I a promic̀e, Gabrielle Marotte, avait vingt ans el était la fille d'une ancienne ouvrière en linge avec qui Willemin vivait depuis plusicurs amnécs"s. Lille avait dû être l'élève de l'artiste puisqu'elle savait dessiner et peut-être même graver ${ }^{(i 0}$. Il l'adopta cn 1831 , puis lui "vendil" en 1832 scs dessins et les planches gravées, pour évitcr sams donte des droits de succession qui l'auraicm obligée à s'en séparer.

Mais l’aide la plus puissante luu colle d'Anclré Poltier, bibliothécairc à Rouentis. Il commaissait Willemin clepuis longtemps, admirail son ceuvre ct roulait la voir achevée. Il ramcula les anciens amis de l'artiste et leur demanda lcur collaboralion pour les dernières planches auxquelles il participa arec un beau lalent de graveur amaleur. ${ }^{(62}$. Il obtint de deux grands colleciomneurs d'objets d'art cle la Renaissance, le baron de Monville et Charles Saurageot ${ }^{633}$, l'aunorisation de farre dessiner certaines des pièces les plus intéressantes de leurs cabinets. Enfin, il chercha el obtint quclques subrentions.

Mais les planches n’étaient roujours accompagnées d'aucun texte. En 1806 , Willemin avait promis que l'ouvrage serait "précécté d’un discours sur les arts du dessin en France" et "les planches, accompagnécs d'une notice hismorique". Les souscripteurs attendaient ces dernières avec d'autant plus d'impatience que les sous-litres cles gravures n'émuméraient même: pas tous les objets représentés. Mais Willomin, bon dessinateur, bon graveur, bon amateur d’ar, n'ćtait pas un écrivain ${ }^{64}$; autodidactc, il manquail aussi de commaissances $s^{\text {ti. }}$. 
Il publia en deux fois, en 1825 et en $1827, \mathrm{~m}$ texte de trente-six pages. On y trouve, après la dédicace au duc de Berri, unc Préface, des P'renues à l'appui de la préface, dans lesquelles il cite Dom Plancher et son Histoire de Bourgogne en décrit un trésor trouvé au $\mathrm{xvI}^{\mathrm{c}}$ siècle clans la tombe de l'impératrice Maria; cnfin, dans une Introduction il traite des sceaux ot des bulles. Cet ensemble, aussi ennuveux qu'incohérent, sans aucun rapport arec les illustrations, laissa les lecteurs perplexes et déçus. Cet essai n'eut pas de lendemain et ces feuillets furent rarcment reliés avec le reste de l'ourrage.

André Pottier arait donc tout à faire biti. Il était persuadé, comme il l'écril dans son Avantpropos, que l'histoire de l'art en France par les monuments ne pourrait de longtemps être cntreprise arce succès. L'ćtude des antiquités nationales était trop récente, et le nombre des latits observés trop petit. Les seuls ourrages utiles à ses yeux étaient les monographies et les collections de monuments détachés, la première étape ćtant la reproduction fidèle, de préférence en coulcurs, des œurres d'art, d'ou son admiration pour les estampes de Willemin; depuis vingt ans, d'autres auteurs et artistes araient publić des ourrages sur le Moyen Âge. mais avec une exactitucle bien moindreciz

Après avoir pensé établir une table méthodique des planches. comportant de courtes notes, André Pottier, à délaut parfois de renscignements précis sur les objets représentés, s'efforça "d’en spécifier les caractères particuliers et généraux, cn faire ressortir el en formuler les trpes, de manière à fournir au lecteur, unc suite d'excmples et de définitions applicables à l'intelligence et la détermination de tous les monuments analogues". Nous sommes loin ici des anecdotes pseudo-historiques el des vagues impressions qui accompagnaient (rop) souvent des illustrations. On recommait aujourd'hui les notices de Pottier comme ćlant sourcut le départ des recherches sérieuses sur telle technique ou telle atégorie d'objcts. Sa méthode, sa rigucur font passer un souffle nouveau.

Confrontant texles et images du passécio, il réalisa le plan d'une histoire du costume en France ${ }^{69}$. Il étudia aussi bien les poteries de l'école de $\mathrm{Pa}$ lissy que celles appelées aujourd'hui "SaintPorchaire". Il apportait dans un style clair des faits satisfaisants pour le lecteur, des réflexions poussant à de nouvelles recherches.

L'établissement de la table des planches posa de nombreux problèmes à Pollicrion. L'ordre chronologique, le classement par matières étaient presque impossibles: en trente ans, l'histoirc de l'art du Moven Âge arail fait d'importants progrès. Willemin lui-même avait eu le temps d'évoluer dans ses datations ${ }^{71}$. L'artiste avait souvent groupé sur la même planche des œuvres qu'il voulait rapprocher et qui, après examen, se révélaient d'époques fort diverses. Désireux d'apporter à la comnaissance des amateurs unc grande variété d'objets, il mêlait détails de bas-reliefs, d'enluminures, d'iroires... Pottier, tout cn reconnaissant qu'il n’arriverait jamais à un résultat satisfaisant, classa au mieux les gravures par siè(les ${ }^{i 2}$, ct, à l'intéricur de chacun d'eux, en architecture, sculpture, tombeaux, costumes, armes,

65 Bien qu'entouré d'hommes de l'art, Willemin ignorait complitcment des problèmes tcls que celui des poussées ell archilcoure. Il cocrin dans les Ammales frangaises des arts, des sciences et des lettres (1821). 6o: "Les cathédrales de nos ancicmnes provinces et particuliercment les églises de Périgueux, de Lyon, de Poitiers, sont dans le plus parfait état de conservation, quoiqu'elles datent toutes, au moins. des nouviène et dixiome siècles. I.'s arc boutans employés par les architectes postérieurs à ces siècles, prouvent non pas l'ignorance... mais la timiclitć de ces maîres de l'ouvre." Il accucillaut les idées du moment sur l'origine de l'art occidental: aux XII" et XIV" siccles, "l'architecture participe beaucoup du goût de celle de l'Inde et de IAsic" (Modèle provisoirc de la table des planches, Bibl. Saintc-(incriève, Réscrve)

66 Potticr rapporte seulement qu'il notait sur un exemplaire de lomrage les renseigneınents que Willemin avait pu lui donner clans leurs conversations.

67 Citons ainsi: Camille Bonnard, Costumes des $x I I^{\prime \prime}, x \|^{\prime \prime}$ et $X v^{\prime \prime}$ sièles... (Paris, 1829); Viel-Castel, Collection de costumes, arme's ret merubles... (Paris). Citons aussi les inonographies de monuments et les voyages, dont les plus célèbres sont l'oyages piltoresques "l romantiques dans l'Ancienne France. de Ch. Vodier, J. Tavlor, Alph de Cailleux, à partir de 1820 .

68 Avant-propos, p. 11: "I.es vieux glossaires, les anciens comptes de dépenses, les antiques inventaires de garderobes, lui ont fourni les termes et les qualifications jusquici si peu fixés, si peu prócis; les chronicyueurs, les romanciers, les scrmonnaires, les moralistes cl les satiriques lui ont préscntć des descriptions, des ensembles plus ou moins complets, qu'il s'est appliqué à confronter aux costumes figurés..." Pottier ajoute qu'il a aussi fouillé dans la mine précicuse des livres anglais sur l'art ct l'industric du Moyen $\hat{A g c}$

tig Avant-propos, p. 11. Il publia en 1831 , dans la Gazette littéraire, ses "Recherches sur l'histoire du costume en France".

70 Willemin avait conscillé en 1825 , dans un avis pour la reliure, de supprimer les planches représentant les statues de Childebert (pl. 88) et d'Lltrogothe (pl. 89) (cxćcutées longtemps après lcur mort, elles n'avaient pas d'intérêt pour le costume), celles représentant une dame de la cour de Henri il (pl. 240) et M Mlle de Montpensier (pl. 253) (exécutécs à une échelle trop grande par rapport aux autres gravures) et deux de la cathédralc ('Amiens (pl. 85 et 123) (faites d'après des dessins erronés). Pottier décida de ne pas les rejeter, de peur de fairc, des volumes qui les comprendraient, des raretés bibliophiliques.

71 Publiant en 1807 (pl.113) des détails d'un gémellion de la Bibliothèque impériale, Willemin l'avait daté dı $x^{e}$ au $x^{e}$ siècles. Quelques années plus tard. il en reproduit. l'ensemble (pl. 112) cn lc datant du X 1 II $^{\prime \prime}$ siècle

72 Il divisa cn deux le $\mathrm{x} v \mathrm{I}^{\prime \prime}$ siècle. 
vitraux, meubles, ustensiles, ornements, etc. ${ }^{73}$ Des années passorrent clans cette longuc cumcle. Lourage lut colin terminé en $18399^{\top 1}$.

Si japprécie ce recueil comme source pour les chercheurs d'aujourd'hui, comnaissant micux son histoire je le considere aussi avec respect comme le résultan du narail acharné el dees priations d'un artiste qui se sacrifia pour le lare aboutir. Il est aussi l'émanation de plusieurs génćrations d'amatcurs, ćrudits, architectes, dessinateurs et graveurs, qui aiderent à sa réalisation pour répandre le goûn des antiquités nationales dont ils étaient cux-mômes fervents admiratcurs. Mais les Iomuments fiancais inédits de Willemin ne seraient jamais que des planches en désordec dans un portefeuille, sans lapport de l'intelligence, du travail el de lammitié désintéresséc d'André Potticr.
73 Potticr ajouta, dans des appendices, des estampes vraiment impossibles à classer, comme celles qui avaicont été copiées sur le Recueil des Roys de France de Dutillet.

74 Vicolass-Xavier W'illemin mourut d'une allatgue, le 27 janvier 1833 . Lne coquille dans l'A amt-propos de Pottier le fait mourir en 1839: elle a été recopićc dans différents dictionnaires. limventaire de ses quelques bicns a brûlé en

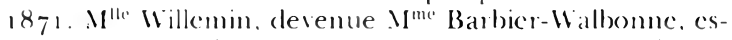
péra longtemps faire un nouveau tirage de l'xeure de son père adoptif. (On trourc mention de lourrage et de ses accessoires (cuivres, dessins, papier, etc.) (lans son contrat de mariage (M.C.. L.xv, 744, 22 mai 1837), dans son testat- mell de 1841 (m.c., cxvill, 1312, 31 mai 1866), l'inventaire aprés dóces de Barbier-Walbonnc (m.c., cxvin, 30 mars 1860$)$. "t dans l'inventaire après décès de (jabriclle, derenue en décembre 186 1 l'ćponse du bancpuier limest-Antoinc Maricot, décédée le 11 mai 1866 (n.c. cion1, 1312, 31 mai 1 866). Ln article de Gućncibault, (lans le journal $1 . l^{\prime}$ nion du 12 férrier $18 j$ ti, relate que les Monuments fremsetis imédets venaient d'aroir une médaille d'encouragement a llexposition universelle er que $\mathbf{I}^{m e}$ Babbicr-lWalbonnc lançait une souscription pour un nouveau dirage. II scmble que celui-ci n'cul pas licu.

\section{Répertoire choisi des planches des Monuments français inédits}

Pl. 11. Détails d'architecturc extraits de divers $\mathrm{ms}$ du $\mathrm{IX}^{\prime \prime}$ siècle. Bibliothèque royale. W'illemin del' et sculp'.

Piliers encadrant cles pages de manuscrits ou des canons d'frangiles.

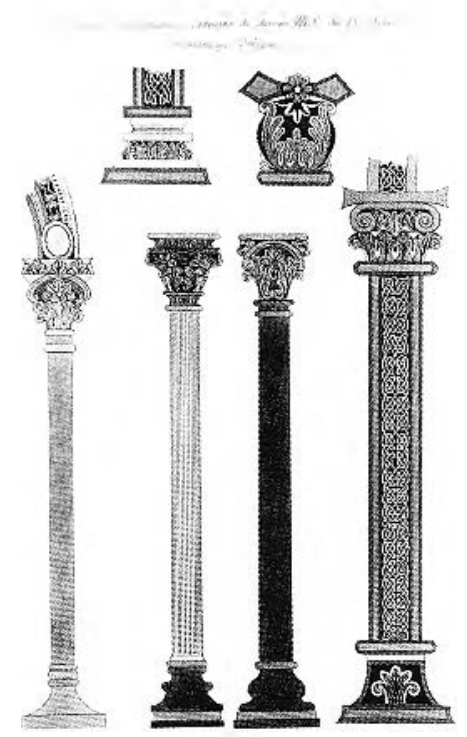

Pl. 14. Trônes et lits grecs extraits d'un us cxécutć en 886. MS n" 510 . Bibliothique du roi. Willemin del' et sculp'

lior brille de toutes parts sur les 45 columinures du manuscrit "d'une magnificence qui ne le cède à aucun autron cl qui contient les oraisons de saint (irćgoire de Narianze. Exemples de meubles civils, à une époque où n'existem guère que des manuscrits religicux.

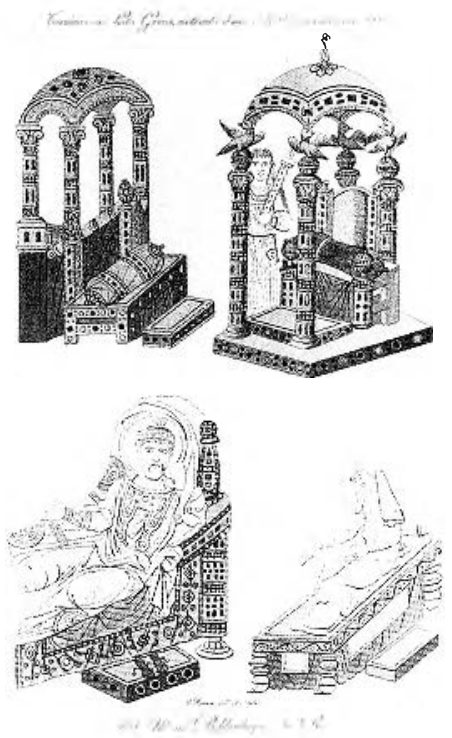

PI. 34. Décoration extéricure du croisillon septentrional de l'église de Saintfilienne de Beaurais I anglois del'. Wil lamin sculp'

"Il n'est peut-être pas hors de propos de faire connaitre, comme un lémoignage de la scrupuleuse conscience que M. Willemin apportait à l'excourion de son ouvrage, qu'il lit trois fois le voyage de Beauvais avectrois antistes différents. pour faire recommencer trois fois le dessin de cette rose. dont il crait mécontont" (Pottier). Le troisiome artiste était soll ami F. H. Langlois. $(1814$.)

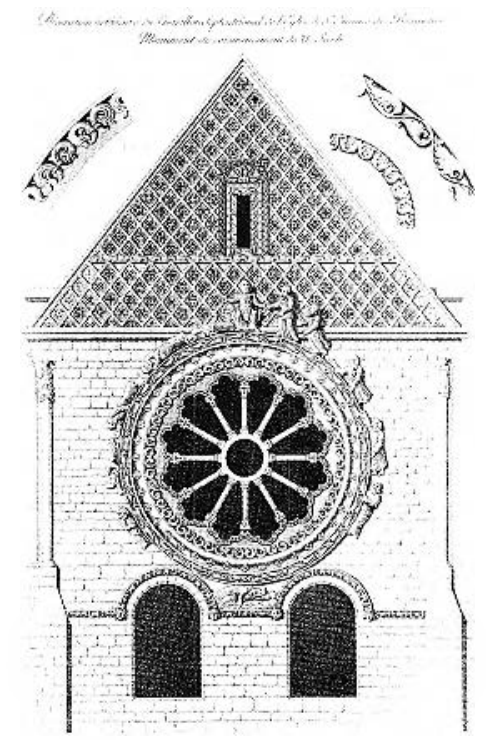




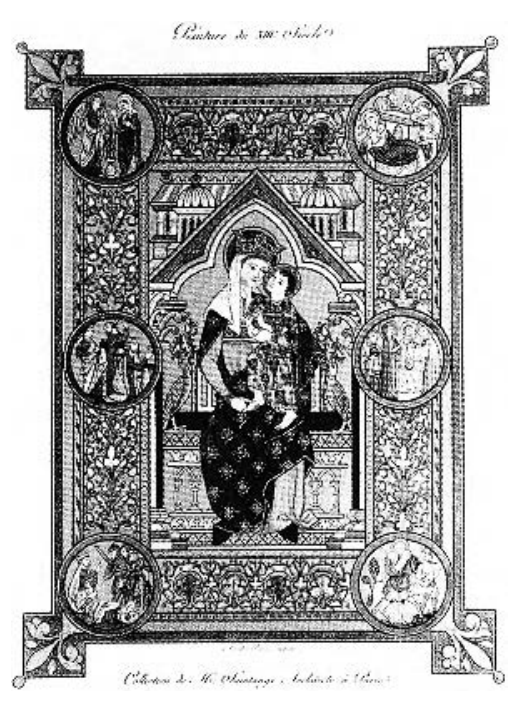

Pl. 99. Peinure du xur" siècle. Collection de Mr. Saminger architecte à Parris. Anćcléc Pérée sculpsit.

l.c fond, jaune ici, est sur l'original, en or bruni. appliqué sur un léger relied.

Pl. 138 . Coiffures du XIV" siècle, tircos du manuscrit de lórence conservé à la Biblionèque de l'Arsenal. (1810.)

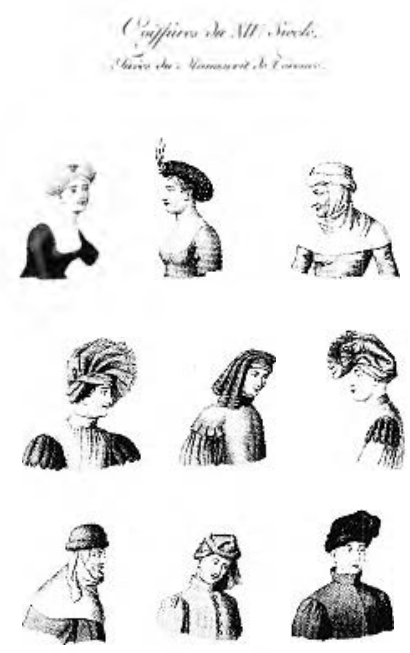

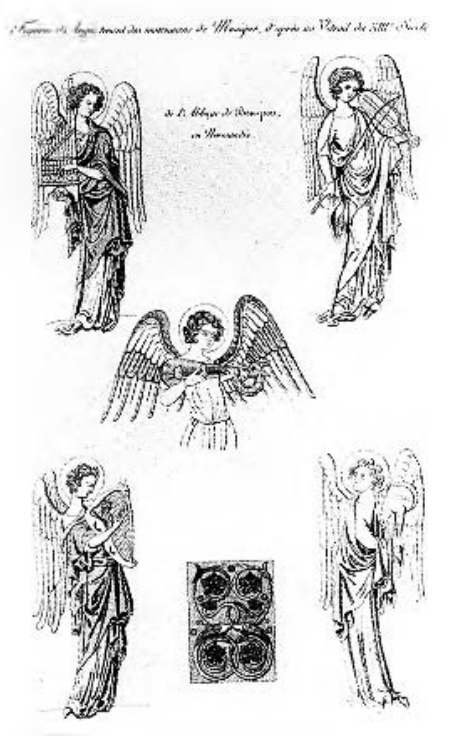

PI. 106. Figures d'anges tenant de's instrumens de musique, d'après un vitrail du Xu" siècle. E. II. Langlois del. Willemin sculp.

Ils viennont de l'abbaye de Bon-Pon, près de Pont-l'Arehe, pars natal de I anglois. Celle abbaye, aujourd'hui détruite. subsistail en grande partic au debur du xIx" siecle. Ces anges som importants pour l'étude des instruments de musique au xiv" siècle, date que préférait Willemin. Lornement, d'aprós A l.cnoir, étail tiré d'un tableau peint sur bois à l'eau d'oxuf, qui représentail Philippe le Bel sur son trone et qui decorair jadlis l'une des salles du Palais de justice de Paris (Atlas, 35) $)(1817$.
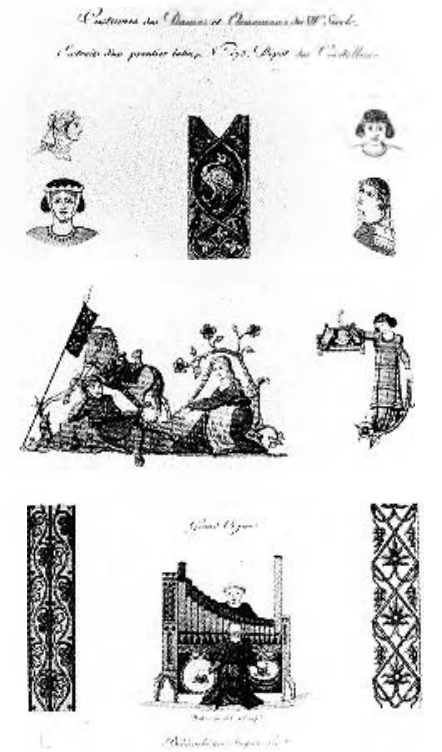

11. 133. Costumes des dancs ct orne ments dlu XIV" siècle, extraits d'un psautier latin, 1" 175, Dépôt des Corcleliers. Bibliothèue impériale. Willemin del et sculp.

Le dépot des Cordeliers était un dépôt rovolutionnaire de livres saisis. Willemin 1 avait dessiné cos conluminures avant que le psautier n'outre à la $\mathrm{Bi}$ bliothèguc impériale. Potticr datait ces miniatures de la fin du xul" siècle à causc de l'armure du chevalier. Cette page est un bon exemple des représentations multiples que Willomin pourait accumuler sur une scule planche. (1814.)
PI. 166. Costumes civils de la fin du xive siècle. Fxtraits de deux paires d'lleures manuscrites de la Bibliothèque royale Heures de 1390. A. Pérée del' ct sculp'.

Pour Willemin, le persomnage en haut à gauche clatit le roi René Celui-ci, mort en 1480 , ne pouvait pas figurer dans des heures de 1390. Les autres personnages ont des costumes du règne de Charles v'. $(1827$. 
Pl. 170. Peinture du $x r^{\text {" }}$ siòcle représentant une halle couverte. Manuscrit grand in-lolio. Bibliothoque de la viile de Rouen. Willemin del' et sculp'. Pérée sculp)

lince de la maduction francaise des Ellhiques el des Politiques d'Aristote par Nicolas ()resmc, dédiée à (harles $v$. Cette copic du second licrs du xve siècle contient un chapitre tratant du commerce. ()n a representé, d'un côté un orferre. de lautre un marchand d'étoffes. (1825)

Pl. 176. Cartes du xu" siècle exécutcos pour le roi Charles vi par Jacquemin Grigonneur, peintre de la cour. Cabinet des dessins et estampes de la Bibliothèque du roi. Willemin delt et sculpt.

C"est un jeu de taross incomples d'un luxe royal

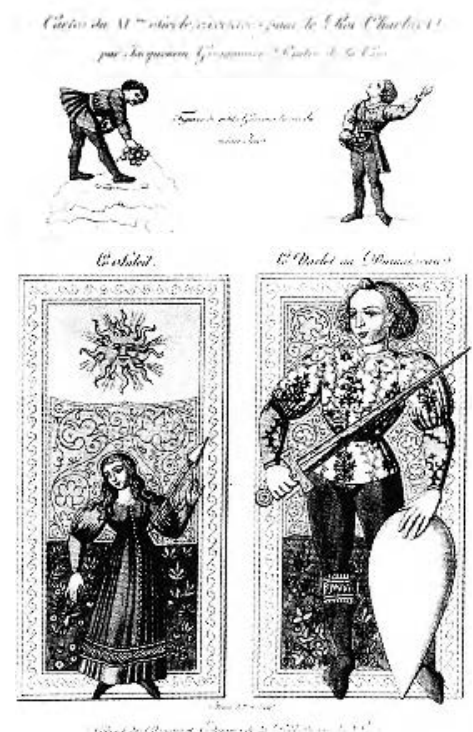

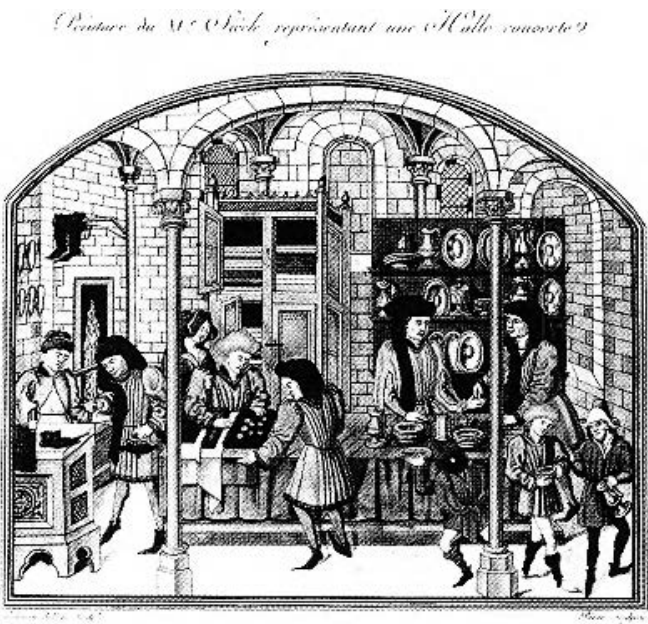

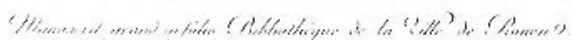

PI. 216 . Encadroment de page, présentant divers meubles et ustensiles de toiletce du xu" siècke. Bibliothèque de l'Arsenal. lurmeau del'. Amílée Pórée sculp's.

Au débur du xvi" siccle less encadrements de pages ne sont plus seukment vegclaux. Celui-ci, que la gravurce a reproduit a une plus grande cohelle, représente des bijoux el objets précicux sur des tablettes. Le milieu de page réserve pour le texte contient une tapisserie ou un cuir cloré, coppié clans un Aristote ale Rouen. (1839)

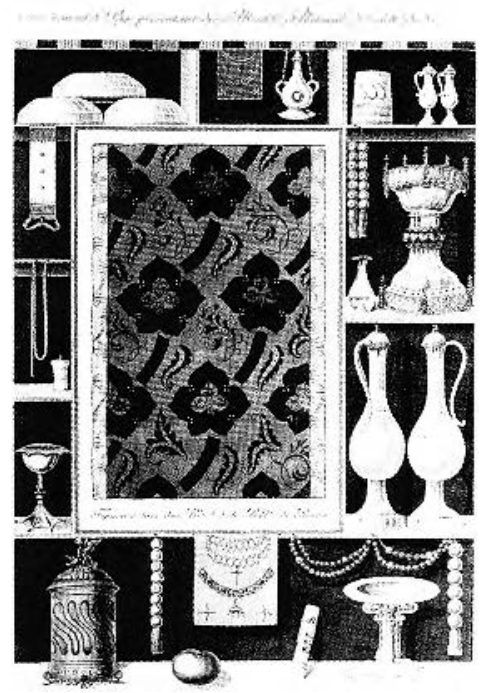

Pl. 231. Maison en bois du Xv" siècle siluce rue des postes à Cacn. Jolimont del'. Fragment d'une porte de SaintMarcel a Saint-1)enis. Fous Debret (lel'. A Pére sculpsit. Détails du palais de justice à Rouen. [O mements] Pugens del'.

la plupart de ces monuments ont disparu. Ia partie représentéc du palais de justice de Rouen, construte en 1493. a beaucoup souffere de la demière gucrere.

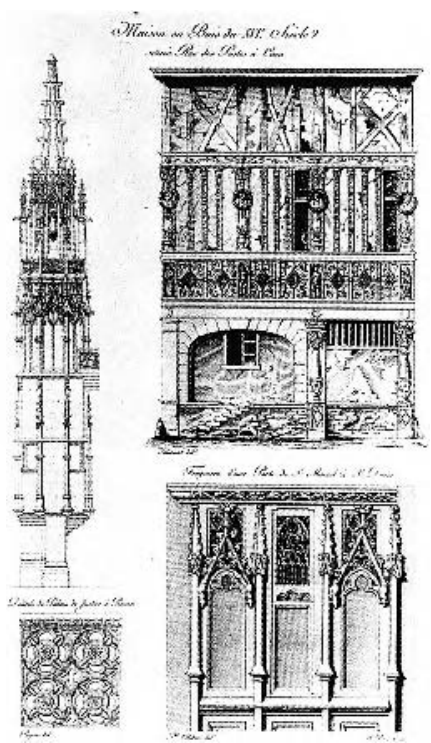




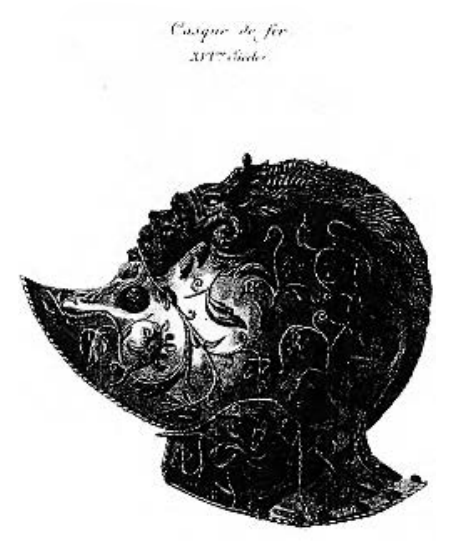

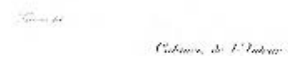

Pl. 25j. Casque de fer. xirt" siècle (Cabinol de lauteur. Thumcau del'. Rogues sculp)

Cicte bourguignolle de G. P'. Negroli, passée daus la collection Révoil an momen ou Willemin dur disperser son cabinct, est entréc an Muséc du lourre on $1828 .(1806$.

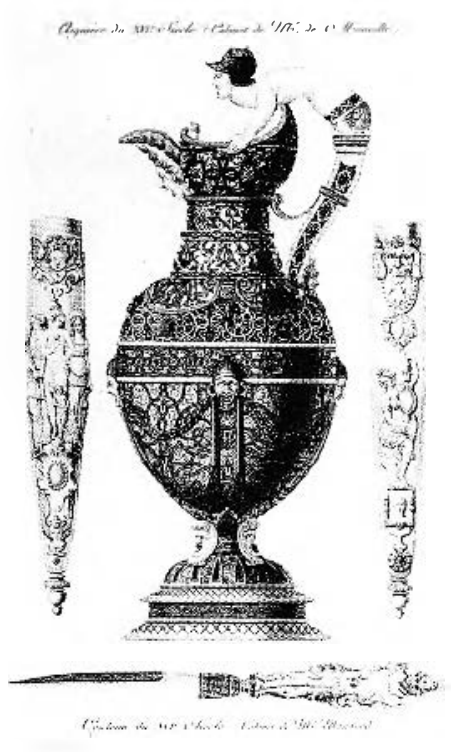

Pl. 289 . Aiguiere du xvi" siocle (Cabinet de Mr de Momille). Couteau du Xvi" siecle (Cabinet de Mr Mansard). (;'" Willemin 'l Langlois del'. Amedee P'érée s(ulp)

(Voir Appendice I.) Latiguicre en Saint-Porchaire est aujourd'hui au Muséc de (leveland. (1839.)

\section{APPENDICE I}

Collections et collectionneurs des objets d'art représentés dans les Monuments /rançais inédits

Willemin, puis Pouter, ont apporté beaucoup) de soin au choix des objets à représenter, quilis ont sélectionnés selon des critères de qualité et de variéré. Ils les ont dessinés ou fait dessiner lans plusieurs sortes de fonds.

\section{A - Collections nationales de Paris}

Bibliothèque impériale, puis royale (aujourd'hui nationale). (abinct des manuscrits: clétails de reliures du psauticr de (harles le Chauve (pl. 7) et d'un évangeliaire de la Santc(Chapelle (pl. 143). - Cabinct des médailles et antiques: ivoires (pl. 18, 4o), armes (pl. 257-260), trone de I)agobert (pl. 1). gémcllion ( pl.112, 113).

Husce Charles $x$ (aujourd'hui Loure): detail du religuaire (lu bras de Charlemagne (pl. 40).

Musée d'artillerie (aujourd'hui Musée de l'Armée): (pl. 178. $25(6,261)$

\section{$B$ - Province}

Bayeux: broderic de lat reine Mathilde (pl 43). Beauvais: bannicre de Jeanne Hachctte (pl.177). Dijon (musée): couteau de Philippe le Bon (pl. 179). Troyes (cathédrale, trósor): iroire (pl. 17), aumonicice (pl. 114)

(: - Collection de Willemin

Voir supra, n. 42 ; ćgalement, réchaud portatif (pl. 215). Il ne (o)pla que dessins el columinures chez ses amis Langlois ot Lenoir; ce dernicr publiait d'ailleurs lui-même des objets de soll cabinet.
1) - Collections des premiers amateurs d'Antiquités nationales A l'index des collectionneurs dont on lit les noms sur les planches des Monuments français inédits, j'ajouterai quelques détails biographiques sans essayer d'épuiser le sujet que jo développerai dans un ouvrage ultérieur. Pour les objets, j'insisterai seulement sur les passages de collection à collection dans l'entourage de Willemin, comme je l'ai déjà fail dans la note $4^{2}$. ()n remarque facilement que nombre de ces ocures d'art sont contrécs au Louvrc, ou ont appartenu à de célc̀bres collections.

Wr de Clermont, all Mans

C.ermon' (Iouis-Gaspard-joseph (le), dit "le comte de Clermont-(Gallerande" (Andolshcim, 17 avril 1744 - Château de la Piltière, près du Mans, 28 mai 1837), officicr jusqu'en 1785 . Son inventaire après décès ayant disparu, on peut hésiter entre lui ct son fils. Adolphe-Armand-Louis-(Gaspard de Clermont-Gallerande (Le Mans, 4 décembre 1798 - lec Châlet, près (lermont, 20 novembre 186i3).

Pl. 41. Tan "n itoire (Victoria and Albert Muscum, $37^{2-}$ 1871 ). Contrairement à ce qu'ćcrit Pottier, nous avons là un tau dont plusicurs partics manquent. Vente Clermont, 18 avril $1864,11^{\circ} 33$, acheté $805^{-1}$ par Ayers.

.17r Crochard, is Cihartres

Sans avoir de preure décisive, je pense quil s'agit de CROCHART (PictTe), propriétaire, mort à Chartres à l'âge de 55 ans lc 21 aôt 1824 , fils de: Pierre-Alexandre Crochart, notaire. Ses neveux firent disperser ses biens immobiliers dans une ventc par le ministère de Hue, à Chartres le $13 \mathrm{scptembre}$ 1824 .

Pl. 30. Crosse de Ragenfroi, dite aujourd'hui "de Wilclmus" (Florence, Bargello, 622). Est passée dans les collections Douce. Merrick et Carrand. 
W' Griveraull, à Paris

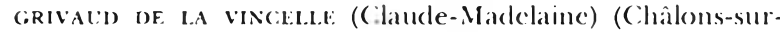
Marne. 3 septembre $17(62$ - Paris, 4 dócombre 1819$)$. Il ajouta a son nom de Grivaud celui de son épouse, M"lt" de la V'incelle. fille naturelle, mais recommue d'fonoré, prince de Monaco. fonctionnaire du Sinat sous l'Empire, il mourut garde des archives de la (hambre des Pairs. Il publia de nombreux ouvages d'érudition concernant essentiellement des monuments de l'Antiquitć. II s'interessait pourtant aul Moyen Age. Plusieurs muméros de la vente de son (abince (2 1 avril 1820) en font preure. Malheureusement "un nombre asse\% considérable d'objets" du. Moven Age ne furent pas decrits (n" 29)) ; il ni arait pas cu d'insontaire après décès.

Pl. 72. Plaque d'émail: Méraclius et un séraphin (Nantes, Musco Dobréc). Cot ćmail mosan faisait partic d'une croix (reconstituce à l'exposition de Stungart, 1977, Dir Zeril der Stunfer. pl. 345 du cataloguc). (collection Saint-Morvs; achetéc pan Griatud avant la vente Saint-Mons du 26 janvier 1818 ; vonte Germeau du 4 mai 1868 , acquise $310^{\prime}$ par Vannheim pour Dobrec

Crosse de cuireve: appartenait-clle aussi i la collection Grivaud? (ene crossc a l'apparence curange n'a pu etre localisce.

Pl. 111. Gémellion. Voir à comte de Maills.

Wr le comente de Mally, pair de Franer

Mall.y (Adrien-Joseph-Augustin-Amalrice comte de) (Paris 19 fóvicr $179^{2}$ - Château de la Roche-Wailly |Sartho|, , juiller 1878). Officier sous l'Fmpire puis sous la Restauration. pair de France en 1815 , atede de camp des dues de Berry el de Bordeaux. Les pièces de sa collection, exposées parfois au XIX" siècle, sont restées dans sa famille sauf les premieres citces.

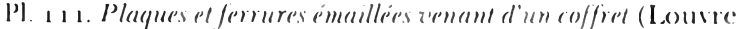
OA-10889, contréces ( $\left.11199_{1}\right)$

Gimellion. Collection Husson, de Sedan; puis collection (irivalud de la V'incelle qui l'a public dans son Rerueril de momumenes. antiques. (Paris, 18,7), 11, 316, pl. 39). Il ful acheté 231 i lat rente apres son décés par le marchand 1)aval (21 avril 1820 . 11" 280$)$.

Pl. 146 . Exper dite d'Edonard III

Ir. Mansard, is Brantelas

Massard (Pierre-Alexandre), né a Beaurais le 29 octobre 1796 . negeciant en gros décoffes (1823). puis de fer (1835). épousa (1821) la fille d'un orféve. P.-A. Mansard, "négociant ct amateur d'objets d'art ot d'antiçutés". servil d'expert pour l'inventaire après déces de Provost, à Bresles en 1833.

Pl. 108 . Email décoré de quatrefenilles, représerentane des monstres "ldes perrogures (fin Xut"). Non localise Il vient sans doute de lal même ceurre que les plaques conscrices au Muscum of line Arts de Boston (48.1320.1). II décotait une reliure an $x x^{\prime}$ siècle.

Pl. sog. Détail d'un gémellion. Non localisé.

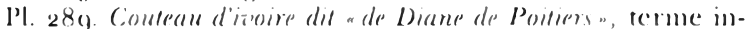
venté par Langlois. Dibelin $(11,493)$ anait achece en 1818 a Willemin un dessin de langlois representant ce coutcan ot

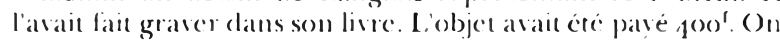
le renoure, estimé 2oot, dans linventaire après décés de Provost. de Bresles (1 3 mai 1 8.33), puis (he\% Mansard. A la vente Debruge-Duménil (23 janvier $\left.185^{\circ}\right), n^{\circ} 176$ ), il est acheté 2000 par un marchand. Linfin, enrichi de diamants, il est acquis pour $17000^{\prime}$ à la vente Spitar (1 7 arril 1893)

\section{Mr de Momidle, Mr Inemonialle}

MoNviLLe (Hippolyte Boissel., baron Boissel de Monville) (12 decombre 1794 - Paris, 2 lévier 1873 ). Il épousa con 1823 lonise de Hontebello, fille du marechal lannes, dont il se sćpara apress en avoir cu une lille II posscelait des filatures pres de Rouen el ćtait proprictaire terrien. Il réunit plusicurs collecrions successives el les dispersa en ventes publigucs. Vous retrouvons dans la premierc ( 7 mars 1837 ) les objers qui venaient d'être gravés dauns les Monuments frangąis inédits. Le cataloguc y fait référence. Sous ke Scond kmpire, Monville servit de mandataire aux Rothschild pour de nombreux achats. 11 mourut ruince.

Pl. 262. Bonclier (n"163 de la rente). I ithographic plus lated

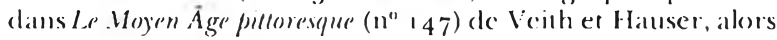
qu'il appartenait alu comte (olbert.

Pl. 264. Fepre d'Henri $N^{\circ}\left(\mathrm{n}^{\prime \prime} 167\right)$. Rapportée de Vieme par Percs, clle fut achetes ares toute la collection de colui-ci con 1825 par Durand en revendue le 18 janvier 1830 (11"66)

Pl. 271. Tapissorie de cuir

Pl. 277 . Dressoir (1"211). I.ithographic consuite dans les Meu-

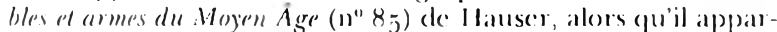
enait a la collection Baron; vente Baron du 19 janvice 1846 , 11 "477, acheté $621^{\prime}$ par Berthon jeune.

l'armiraux sculptés de têters (11"222)

Derux charses plianter. On les retrouse dans la collection Spitzer (catalogue de 1891,111 , meubles, $11^{115} 63$ et 64).

Pl. 283. (ile derqurbuse (11"172). Peut-être est-ce une de colles acherees par Provost a la vente Samt-Norys (n" 248). fille appartint consuite a la collection Sommesson (vente du 24 janvier 1848,110232$)$

Pl. 288. Fimail primt: Ia * (iramalique" (11" 83. vendu 4510 ). II a appartemu ensuite à Anthomy de Rothschild. II cst passé en V'ente le z mai rajt (V'ente de la lendation Vorton Simon. l'arke Bernet, 21000 dollars)

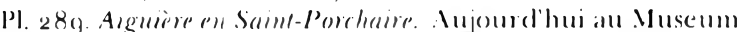
of tur de (ideveland. apres avoir appartenu a la collection Amhonr de Rothschild.

I'l 110. Címellion. V'oir is Provost.

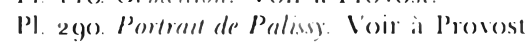

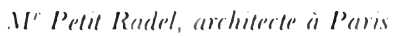

PETTT-RADrt. (I.ouis-Fiangois) (22 juilled 1740 - Paris, 7 noscmbre 18,8). Architecte (élebre aujourd'hui concore pour avoir expose alu Salon de l'an vill, arec sa notice explicative.

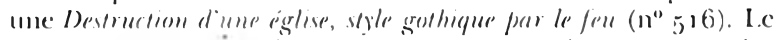
goût du Voyen ige lui étant vemu plus tard. on trouse plusicurs objers de cotre période dans sal collection.

Pl. 107. (rome limousme. Aujourdhui au Rijksmuscum d Amsterdam. N"220 de la vonte Perit-Radel du 8 févier 181 g. Hille passal consuite par la collection Pourtaless le Muséc de firmitage er une collection privée ditusterdan (J. J.

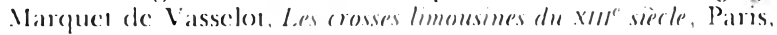
$\left.1941,11 " 3 \cdot p^{1}\right)$ 1)

lixide limousine. Non localisce.

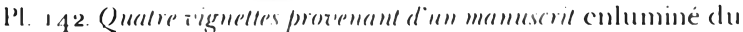
xur" siecle (On retrowe trois dentre clles aves une autre de mêne origine (anns la vente Debruge-Duménil du 23 janvier $1850,11^{\prime \prime} 45^{2}\left(1^{\prime \prime}\right)$, achetcur (ombrouse, 70', et dans la vente (combrouse du 14 mars 1859.9 .11164

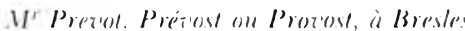

PRovost (Toussant-Naric-Amable) (23 jamsice 1761 - Bresles [()isc] - 2 mars 1833). Arocal an Parlement. procureur du roi con la maitrise des caux el forêts de liance an département de (ilermont-en-Beauraisis, ce motable cultivé accueillit arec joic la Rivolunion mais due se cacher pendant la lerreur. Il collectionna surtout des antiques, des pierres gravées, mais aussi des émaux, ivoires, medailles.

Pl. 7. Plaque mosume: trois apôtres à mi-corps. Elle a appartcrut consuite à la collection (Chalandon (Catalogue de l'exposition

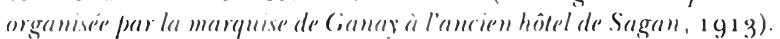
Fille était ches Wertheimer, on 1954

Pl. 1 10. Cómellion (Victoria and Albert Muscum, 574-1910). Il a appattem an baron de Momille (vente 7 mars 1837 , $11^{\prime \prime} 8_{1}$ ). puis a Debruge-1)uménil, Scillicre et Desmoucs

Pl. 254 Casque. Si tous les objets représentés sur la planche vicuncont de la collection Provost, il a peut-cotre appartenu aupatalamt a Saint-Morss.

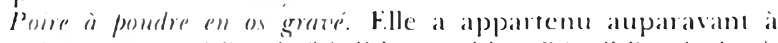
Sam-Morrs qui l'avait lait lithographier. Révoil l'anait dessinoc (Cabinct des dessins du lourre. A. I.cnoir, R.F. 5280, 1. 11, $[0 ; 7)$.

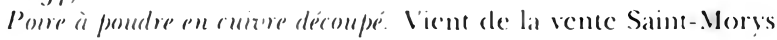
(11" 2,46$)$ 
Pl. 2 go. Pontrail dit "de Bermard Palissy" en poterie vernisséc. Il a sans doute appartenu à Alexandre Lenoir, d'après un reşu du 19 florial an x. Il appartint ensuite an baron de Monville. puis à sir Anthony de Rothschild.

Pl. 289. Contedu de Diane de Poitiers. Voir à Mansard

II' Rogé, à Paris

roger (Salomon-I,ouis, baron) (Genève, 29 novembre $177_{j}$ Paris. 4 oc(ob)re 18,1 ) possédait un riche cabinel comprenam quelques objets médiévaux de qualicé.

Pl. 41. Ciosse d'izome d'Yros de Chartres (Florence, Bargello). Avant la Révolution a l'abbaye Saint-Quentin de Beauvais, puis collection (ammbry, Saint-Morys (ventc, n" 108 ; achercur Barthélémy, $210^{\prime}$ ), collection Roger (vente du 27 janvier 1842 , n" 45; acheteur (arraud, 180')

$M^{\prime}$ de Sam--Morys, maire à Hondainzrille

SAINT-MORYS (Charles-Filieme de BOURGEVIN VIAI.ART, comte de Saint-Morys) (17 janvier 1772 - Paris - 21 juillel 1817) Voir note 10. Son pore perdit pendant la Rérolution sa fortunc et sa très grande collection de dessins. Lui-même, revenu d'ćmigration en 1802 , lit dessincr les églises en destruction. Il cut la plus grande collection d'objets d'art du Moyen Agc du débu du $x_{1 x} x^{\prime}$ siècle. Il fü tué dans un duel cn 1817. Seulement deux planches des Monuments inédits portent son nom commo propriétaire de dessins (pl. 244 el 263). Mais nous retrouvons des objets de sa collection dans les collections Grivaud, Provost, Roger, Saluvageot.

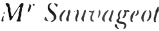

SaUVAgeot (Alexandre-(harles) (novembre 178, - Paris 30 mars 1860$)$. Second violon à l'orchestre de l'opéra à partir de 1800 , il cumula vers 1810 cet emploi avec un antre dans les Douanes. En môme temps, il constituait unc importante collection d'objets d'art (le la Renaissance qüil domma au lourro en 1858. Vommé conservateur honoraire, logé an loure, il mourut peu apres.

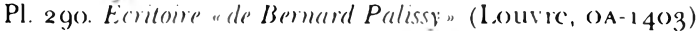

PI. 291. Plal orale: Ia "r)mphe de Fontaineblean (Iourac. $\mathrm{OA}-1341)$

Pl. 280. Miron du XV" siecle (Louvre, OA-359).

Mouchelles (I.ouvre, oA-279), déposées au Musée de (iluny le 14 décémbre 1936

Clef du $x^{\prime} \mathbf{r}^{\prime}$ siócle (I.ouvre, oA-871), déposéc all Muséc de Cluny le 14 déccmbre 1936 .

Peigne. Il n'a pas tail partie du don Saurageon an Muséc du Loure

Pl. 281. Souflel an bois sculpté (Lourre, OA-407)

Voir aussi note 43 el Van Hoorn.

.$M$ de Senomnes

SENONNES (Alexandre de la MOTIE-BARACE, vicomte le) (Scnomnes [Mayemne], 3 juillel 1781 - Paris, 21 mars 1840). Fils d'un noble collectionncur, mort sur l'échafaud. Ayant récupéré une parlie de sa fortume, il voyagea en Italic. A la Restauration, il rentra en france et devint secrétairc de la $(\mathrm{ham}) \mathrm{he}$ du roi, membre de l'Institut, secrétaire général des Musćcs royaux (1816-1821), secrétaire général de la Maison du roi, maître des requêtes, intendant de la liste civile. Il fut ruinć par la révolution de 1830 .

PI. 276. Drux chaives composites. Elles apparticmncml alljourd'hui au Muscé de Cluny, mais ont été démontées (Catatlogue Dusommerard, 1509 et 1510 . Ins. 152). A. Dusommerard, après les avoir acquises, les avait publiées dans Less Arts du. Mojen Âge. Allas, chap. XII. pl. vi

Meuble du XVI" viecle. Sans doute celui que l'on troure en 1828 dans l'inventairc après décès de la première $\mathrm{M}^{m *}$ de Scnomnes, celle dont Ingres fit le portrait aujourd'hui au Musée de Nantes: "Un cabinct antique avec cariatides, figures ct ornements sculptés en bois de vigne..."

Mr Turbé à Se'us

Tarbé. Millin, lors de son Voyage dans les départemens du midi de

la France (Paris, $1807-1811,57$ ), était passé par Sens où. comme plus tard Willemin, il avait eu recours à $M$. Tabc: "plusieurs de ses frècs exercent, dans les villes voisincs, l'état. d'imprimeur el le libraire, qui étoil celui de leur pere. M. l'arbé réunil dans Sens ces deux professions. ()n trouve dans l'Anmunire du défarlement qưil rédige. des notices très-bicn faites: il a du aroir sans prétention, une bontć et une obligeance extrimc."

Pl. 29 et 68, représcntant denux dessins de la collection T'arbé : la crosse d'Atalde cr lo tombeau d'Henri Sanglier

M' le baron de Van Hoorn, à Paris

VAN HOORN VAN VI.OOSWICK (Pierre-Nicolas, barom) (Amsterdam, 27 mars 1742 - Paris, 5 janvier 1809). Hollandais, amateur d'art, il sćjomma longtemps en Italic où il collectionma surtout des pierres gravécs. Il se fixa à Paris pondant la Révolution ex profita de l'afllux des ouvres d'art sur le marché pour se constimce $11 n$ riche cabinet. On y trouvait de tros beaux antiques, de nombreux vases de pierres dures, mais aussi un ivoirc gothique (n" 512 de sa vente après décès du 22 novembre 1809), wne coupc à courercle en Saint-Porchairc (n" j63). Il ćlail lic à Alexandre Lenoir qui fut un de ses exécuteurs testamomaires.

Pl. 28j. Ciniller orienlale à décor filigrané. Entrée au lowro (oA-635) avec la collection Saurageot, déposćc an Musće de Cluny le 14 deccmbre 1936

Cimiller is triple fin (aver fourchette et stylet). A. Potticr écrit quielle appartenait à la collection Sauvageot; clle n'est pourtant pas contrec au lourre.

Comporr. Des 1807 . Millin, dans le Magasin encyclopédique, anait fait remarpucr que cot objet venait des Indes. L'erreur ful pourtant reprisc dans la vente Van Hoorn (n" 77).

Pl. 42. I.e diplyque dirone de Becunrais, aujourd'hui an Muscec de Clum (Cataloguc 1)usommerard 1041-1042) est reprocluit sans nom de proprićlairc. ()n ignore à qui il a appartenu contre la mort de (ambry et son acquisition par Dusommerard. Il est gravé inversé

\section{APPENDICE II}

Dessinateurs el graveurs des Monuments frangais inédils

J'ai reporté ici, par ordre alphabétique, tous les noms d'artistes trouves on bas des planches, que ces dessinatcurs ct graveurs aient travaillé pour les.Momuments frangais médus. on que leurs dessins, leurs estampes aient été recopićs.

Arnaud

Bridgens (Richard). Brongniart (Alexandre)

Camilli. Chaills. (jucton

Debret (Francois). D)(lscnbach. Dubut.

Gabriel. (ianncrey (iannerey (Auguste). Ganncrey (l. F.). Gau. Géraud (ou (jiraud).

Imbard (lit. l. .)

Jazet. Jeanjean. Jolimont (cle). Joron. Juvenell ( $F$.).

I anglois (F. H.). I anglois (Mlle). Le Bas (H.), architecte. I.e Fevre. Le Maître. I.c Maîtrc (Mme).

Malpiece. Marone (Alphonse). Martin. Morret.

Normand fils.

Pauquet. Pecharman (C..)*. Percier. Pérée. P'́réc (Amćdćc). Pottier (André). Pugcus. Pugin.

Régnier (F.). Révoil (P.). Roques

Saint-Ange. Stothard

Thierry. Thiollet. Tummeau.

Underwood

Van Clemputte. Van Saerdam (Pitre). Vauzelle. Vćron. Vìze (Ch. de)*

Willemin (Gabrielle). Willemin (Nicolas-Xavicr).

* Charles de Vicre Pecharman signail de l'un ou l'aurre de ses noms. 


\section{APPENIOICI: III \\ Datation des planches des Monuments fronçais inédits}

On ne sait ou se Irouvent les dessins préparatoires de Willemin et de ses collaboratcurs. et si même ils cxistent encore. Certains d'entre cux, qui avaicnt ćtć exposés, portaient une date. On en voit rarement dans la lettre des gravures. ("est donc les dates de parution des planches que jai entrepris de rechercher

Aucun des quatorze exemplaires des. Monuments français medits que j'ai pu exammer à Paris et à Rouen ne porte sur ses planches d'indication manuscrite concernant la date de leur livraison. Plusieurs des bibliothèques se trouvant sur la liste des souscripteurs ont brûlé (Beaurais. Chartres); diatres ne possèdent plus l'ouvrage de Willemin (Chambre des députćs); aucune. à ma comnaissance, ne garde des archives anciennes assez. détaillées pour indiquer le sujet des gravures.

\section{(C)MPOSITION DFS RF(CLFIIS}

lreizc des quatorze exemplaires ćludiés comportcnt: Arant-propos a notices d'A. Pottier, table des souscripteurs. table des matières. 2 pages de titre et $3(0)$ planches gravées. Celui du Sénat (anciennc (hambre des pairs) n'a quic 23 plan(he's

(On a relié en plus, dans certains de ces recucils: Ie titre de 1825 avec vignette con couleur, le texte de Willemin de 18251827 . des prospectus. Celui de la Bibliothèque municipale de Rouen est le scul à inclure unc première liste de souscripteurs (cntre 1821 (1 1824). La réscrve de la Bibliothèque SainteGeneviève possède, dans le fonds (;uénćbault, une lable provisoire des planches (vers 1815 ).

Sur les zo3 planches que peul comprendre un recueil, on en a peint conviron 185 . tout ou partie (il n'y a parfois qu'un trait de coulcur). Je ne comnais pas d'exemplaire non peint. I.enluminure de colui des Arts décoratifs a été interrompue: il n'y a généralement quiune coulcur par planche, ce qui laisscrait supposer une certaine division du travail. Je ne connatis aucun des exemplaires sur vélin annonces par Willemin dans ses rćclames. Il a cxisté des volumes plus luxueux, exćcutés par excmple pour des collectionneurs ayant permis de reproduire des objets de leurs cabinets. On lit dans le catalogue de la première vente du baron de $110 n v i l l e ~(7$ mars 1837): " 263 . Louvrage de Villemain [sic], avec plusieurs planches coloriées qui ne se trouvent pas dans les exemplaires ordinaires."

\section{SOL:R(AS POL:R LA DATATION}

Elles doivent toutcs être discutces; on y trouve lacunes el crreurs. Je suis arrivée à dater 129 planches de façon précise. Les autres ne peuvent l'être quapproximativement et par recoupements du recueil lui-même, des sources manuscrites el des sources imprimées.

\section{Le recueil hui-même}

Nous avons vu plus haut qu'à part les pages de titre, il contient peu de dates. "Bibliothèque impóriale". "Bibliothèque royale", paraissent des indications précises, permettant d'englober une période. Ainsi unc gravure faite d'après un manuscrit de la "Bibliothèque de Monsieur" devrait avoir été exécutée entre 1816 ct 1824 ; pourtant Willemin, emporté par l'habitude, fait porter cette mention à une estampe de 1828 (pl. 217 )

I a lettre des planches, bien que calligraphiéc: avec soin. est souvent source d'erreurs. Les noms propres sont, à plusicurs reprises, mal orthographiés. I. titre ne porte sourent que sur une parlie des objets reproduits, sans que l'on sache exactemont lesquels. On publie des dessins, faits quelqucs années auparavant, sans modifier toujours lc texte qui les accompagnc. Par contre, une gravure copiée dans un livre ne peut être que postérieure à la parution de celui-ci.
L.es noms d'artistes fournissent certains éléments: un clessin de Civelon ne peut avoir étć fait après 1831 , date de sa mort; a Gabrielle W'illemin ne s'appelle ainsi qu'après avoir été adoptco en 1831 . On sent aussi, en lisant les notices de Pottier. que cortaines planches sont antérieures à sa collaboration (vers 1830). Il y laisse transparaitre son incompréhension devant telle ligende, tel choix, son ignorance du sujet de tcl dérail

Le volume de la bibliothèque du Sénat comporte 23 planches appartonant en général aux demières livarsons. ()n : rrouve pourtant les émaux de Mailly, parus avec le texte de $182 j(p) 1111)$. D'autres. qui detraient y ĉtre. manquent: gravures (l'objels des cabincts Sauvageot (p). 281, 291), Monville (p) $277,288.289$ ), cortains étant signés de $G^{\prime \prime \prime}$ W'illemin (p). 289,291$)$.

\section{Sorurces manuscrites}

Le Dépôt légal était double pour les gravures. (On en gardc trace dans les registres des archives du Cabinet des listampes de la Biblionhèque nationale, dès 1806 , ct dans ceux du secrétariat de la Direction générale de l'Imprimeric et de la l.ibrairic, aujourdhui aux Archives nationales (à partir de 1811). Par ailleurs une erreur dans une póriode troublée où le dćpôt

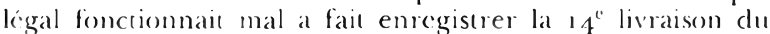
31 aôil 1814 aux Imprimés de la Bibliothèque nationalc. On trouve dans toutes ces archives des lacumes, des erreurs. La 5)" livaaison est un peu détailléc aux Archives nationales: "des éguierres [p]. 289$]$, des intérieurs, des encadrements [pl. 216 ).

\section{Sources imprimies}

Le Magasin encrolopeidique (de 1806 à 1 816), suivi des A muales amoclopédiques (1817-1818), revue dirigéc et rédigéc en partic par Nillin, ne lui survira que quelques mois. Millin. pour soutenir les Momuments franguis imédits, aunonşait les livraisons en indiequant le détail des planches. Mais il oubliail parfois de le faire, ou bien se répétait à plusieurs numéros d'intervalle. les titres quil domnait étaicm souvent vagues, mais ils mont. cu génćral, permis des identifications. Chose curicuse, il arrivait à Millin de citer des estampes (fui n’avaient pas cncore étc portées au Dépôt légal. Ainsi. la $18^{\circ}$ livraison est commentéc en février 1817 dans les Annales encyclopédiques et apparait en juillet dans les registres. Millin en arait-il eu communication a l'avance, ou l'artiste était-il en relard pour accomplir les formalités légales: Dans le doutc, je (hoisis toujours la première clate.

Apres la mort de Millin. c'est le silence presque complet. Les Ammales frangaives des arts, des scionces et des lettres de 1821-1822 citent, dans la $35^{-1}$ livraison, "un saint Louis sous le nom du roi Salomon" (pl. 94). Le Journal général de la litterature de France $(1825), 17$, (1 $(1828), 88$. domne le détail des $36^{\circ}$ ct $44^{\circ}$ livraisolls.

Enfin la lable des matières provisoirc (Bibl. Saintc(ioneriève, réserve) domne tous les citres jusquen 181 jel quelques-uns de 1817. Une planche (35) fora partie d'une livraison de 1825 . Mais dautres ne paraitront jamais. On peut parfois en comprendre la raison; ainsi. Dibdin a acheté en 1818 a Willemin deux dessins de Langlois pour publier dans son ouvrage: (tome il) : le "couteau de Diane de Poitiers" en ivoire ce un plat "de Bernard Palissv". W'illemin qui les arair annoncés dans la table provisoire renonça à les faire paraître. Mais vingt ans après, Pottier fera figurer le coutcau dans la dermiere livarison. 


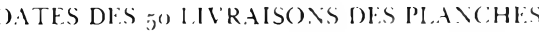

Chaque livarison normale comtient 6 planclies.

1 Dépôt légal, Bibl. nat., list.

2 Dépôt lćgal, Arch. nat.

3 Dépôt légal, Bibl. nat.. Imp.

4. Magasin encoclopédique, puis Annales encrelopédiques.

5 Aunales fiancarises des arts, des scuences et des lettres.

6) Journal géméral de la littérature de France.

\begin{tabular}{|c|c|c|c|}
\hline \multirow[t]{2}{*}{1} & 29 aril $1806^{\prime}$ & $x \times v$ & 22 déc. $1819^{1.2}$ \\
\hline & mai $1800^{-1}$ & $x \times \vee 1$ & 2 fér. $1820^{1 \cdot 2}$ \\
\hline \multirow[t]{2}{*}{ II } & 13 juin $1800^{\prime}$ & $x \times v 11$ & 7 sept. $18.20^{1.2}$ \\
\hline & sept. $1806^{-1}$ & $x \times v i 1$ & 7 sept. $1820^{1.2}$ \\
\hline \multirow[t]{3}{*}{111} & 13 août $1806^{\prime}$ & XXIX & 3 fév. $1821^{\circ}$ \\
\hline & scpt. $1806^{\prime}$ & $x \times x$ & 3 fér $1821^{1}$ \\
\hline & jplanches & \multicolumn{2}{|c|}{ sans muméro de livraison } \\
\hline \multirow[t]{3}{*}{ IV } & 10 arril $1807^{-1}$ & \multicolumn{2}{|c|}{$($ XxIx $0 u x x x)$} \\
\hline & mai $180 \pi^{4}$ & \multicolumn{2}{|r|}{3 fév. $1821^{2}$} \\
\hline & nov. $1807^{4}$ & $\mathrm{XXX1}$ & 30 mars $1821^{1.2}$ \\
\hline \multirow[t]{2}{*}{$v$} & 28 sept. $1807^{\prime}$ & $x \times x 11$ & $200 \mathrm{OCl} \cdot 1821^{1 \cdot 2}$ \\
\hline & Пом. $1807^{-1}$ & $\mathrm{XXXIII}$ & 22 nov. $1821^{1,2}$ \\
\hline \multirow[t]{2}{*}{$\dot{V 1}$} & 18 juill. $1808^{\prime}$ & $\mathrm{XXXIV}$ & 3 jant $1822^{\prime}$ \\
\hline & juill. $1808^{\prime}$ & & 9 jans $1822^{2}$ \\
\hline \multirow[t]{2}{*}{ VII } & 18 juill. $1811^{1.2}$ & $x \times x v$ & 13 fév. $1822^{1.2}$ \\
\hline & scpt. $1810^{4}$ & $x \times x \vee 1$ & 22 déc. $1827^{1}$ \\
\hline \multirow[t]{2}{*}{ VIII } & 18 juill $1811^{1}, 2$ & \multicolumn{2}{|c|}{ "obscrvalion: remis ch } \\
\hline & oct. $1811^{1}$ & & 18250 \\
\hline \multirow[t]{3}{*}{$\mathrm{IX}$} & $17001811 \%$ & & 22 déc $1827^{2}$ \\
\hline & nor. $1811^{-1}$ & & jans $1825^{6}$ \\
\hline & fév. $1812^{4}$ & XXXVII & 22 déc $1827^{\prime}$ \\
\hline \multirow[t]{2}{*}{$x$} & 2 jallv. $1812^{1.2}$ & \multicolumn{2}{|c|}{ "obscrvalion: remis cul } \\
\hline & mars $1812^{4}$ & & 1825 \\
\hline \multirow[t]{2}{*}{$x 1$} & 26 mars $1812^{1.2}$ & & 22 déc $1827^{2}$ \\
\hline & juill. $1812^{4}$ & $\mathrm{XXXVIII}$ & 22 déc $1827^{\prime}$ \\
\hline & 13 juill. $1812^{1}$ & \multicolumn{2}{|c|}{ "obscrvalion: remis (n) } \\
\hline \multicolumn{2}{|c|}{ sans numćro de livraison } & & 18250 \\
\hline & 9 juill. $1812^{2}$ & & 22 déc. $1827^{2}$ \\
\hline & a()ût $1812^{4}$ & $\mathrm{XXX1X}$ & 29 déc. $1827^{1.2}$ \\
\hline & II) $1812^{4}$ & $X L$ & 29 déc. $1827^{1.2}$ \\
\hline $\mathrm{X} \| 1$ & 3 aćc. $1812^{1.2}$ & $X L 1$ & 29 déc $1827^{1.2}$ \\
\hline & $1814^{1}$ & $X L I I$ & 27 déc $1827^{1.2}$ \\
\hline $\mathrm{xIV}$ & $310 \mathrm{OCt} \cdot 1814^{3}$ & XLIII & 27 déc $1827^{1,2}$ \\
\hline & $1814^{4}$ & $X L I V$ & mars $1828^{\circ}$ \\
\hline$x v$ & oct. $1815^{-4}$ & xLV & 24 fév $^{\prime} .1830^{\prime}$ \\
\hline$x \vee I$ & $\begin{array}{l}4 \text { mars } 1815^{1.2} \\
\text { sept. } 1815^{-1}\end{array}$ & sans $\mathrm{nI}$ & $\begin{array}{l}\text { néro de livarison } \\
\left.24 \text { déc. } 183^{2}\right)^{2}\end{array}$ \\
\hline Xvil & 21 déc. $1816^{1,2}$ & sans 111 & méro de livraison \\
\hline xVIII & 18 juill. $1816^{1.2}$ & $X L V I$ & 3 juin $1830^{1.2}$ \\
\hline sans & s numćro de livraison & & 7 planches \\
\hline & fér. $1817^{\prime}$ & XLVII & $80 \mathrm{oct} \cdot 1830^{1.2}$ \\
\hline $\mathrm{xIX}$ & déc. $1817^{\prime}$ & & 4 planches \\
\hline Sans & s numćro de livraison & XLVIII & $10 \mathrm{nor} .1831^{1}$ \\
\hline & 23 déc $181 \%^{2}$ & & 10) $1101.1831^{\circ}$ \\
\hline & déc. $1817^{\prime \prime}$ & sans 11 & néro de livraison \\
\hline$x x$ & janv. $1818^{1}$ & $x \operatorname{LIX}$ & 22 avil $1833^{2}$ \\
\hline & if janv. $1818^{2}$ & & 7 planches \\
\hline & jans $1818^{4}$ & L. & 15 jans $1839^{2}$ \\
\hline $\mathrm{XXI}$ & 4 a)uิ $1818^{1.2}$ & Planches & el rexie. "- 6 ion \\
\hline$x \times 11$ & $\begin{array}{l}\text { fév. } 1819^{1} \\
2 \text { fóv. } 1819^{2}\end{array}$ & $\begin{array}{l}\text { 6] gravi } \\
\text { des é(fu }\end{array}$ & $\begin{array}{l}\text { ces représentaml } \\
\text { (rres, des intci- }\end{array}$ \\
\hline $\mathrm{x} \times 111$ & 20 sepr. $1819^{1, \cdot 2}$ & rieurs, & des encadre- \\
\hline XXIV & 25 sejt $181 \mathrm{~g}^{1.2}$ & ments... & \\
\hline
\end{tabular}

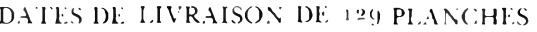

\begin{tabular}{|c|c|c|c|c|c|}
\hline $\begin{array}{l}X^{\prime \prime} \text { de lat } \\
\text { planclee }\end{array}$ & $\begin{array}{l}N^{\prime \prime} \text { de la } \\
\text { livraison }\end{array}$ & $\begin{array}{l}\text { Amuce de } \\
\text { parution }\end{array}$ & $\begin{array}{l}\mathrm{N}^{\prime \prime} \text { de la } \\
\text { planclue }\end{array}$ & $\begin{array}{l}\mathrm{N}^{\prime \prime} \text { de la } \\
\text { livraisont }\end{array}$ & $\begin{array}{l}\text { Année de } \\
\text { partution }\end{array}$ \\
\hline er titre & I & 1806 & $140^{\circ}$ litre & III & 1806 \\
\hline 3"litre & $\mathrm{L}$ & 1839 & $141^{10}$ titre & VIII & 1811 \\
\hline $4^{\circ}$ litre & VIII & 1811 & $142^{\prime \prime}$ litre & $\mathrm{x} I \mathrm{x}$ & 1817 \\
\hline $7^{\prime \prime} \operatorname{titr}$ & I: & 1806 & $143^{\prime \prime}$ litre & IV & 1807 \\
\hline $9^{e} \operatorname{titr}{ }^{\circ}$ & 11 & 1806 & $144^{\circ}$ litre & $x \times x \vee I$ & 1825 \\
\hline $10^{\circ}$ lille & $V^{\prime} I$ & 1808 & $117^{\prime \prime}$ ritre & $\mathrm{XIII}$ & 1812 \\
\hline $15^{\prime \prime}$ iाl & IV & 1807 & $14^{80}$ ille & $v$ & 1807 \\
\hline $16^{\prime \prime}$ tilro & IX & 1812 & $149^{\circ}$ itre & III & 1806 \\
\hline $17^{\circ}$ (ille & $\mathrm{x} I \mathrm{x}$ & 1817 & $160^{\prime \prime}$ litre & III & 1806 \\
\hline $24^{\prime \prime}$ inte & $X X X V I$ & 1825 & $161^{\prime \prime}$ litre & 11 & 1806 \\
\hline $25^{\circ}$ litic & $x V_{I}$ & 1815 & $162^{\circ}$ litre & XII & 1812 \\
\hline $29^{\circ}$ lille & XVIII & 1817 & $164^{\prime \prime}$ litre & $x \times x \vee 1$ & 1825 \\
\hline $34^{\circ \prime}$ titre & $\mathrm{XIV}$ & 1814 & $1666^{\prime \prime}$ itre & $x$ LIV & 1827 \\
\hline $35^{\circ \prime}$ titre & $x \times x \vee I$ & 1825 & 170 litle & $x \times x \vee 1$ & 1825 \\
\hline $3^{6^{\prime \prime}}$ litrc & $\mathrm{xx}$ & 1818 & $182^{\circ}$ litre & XLIV & 1827 \\
\hline $38^{\prime \prime} \operatorname{litr}$ & IX & 1812 & $183^{\circ}$ litre & VII & 1810 \\
\hline $39^{\prime \prime}$ litre & 11 & 1806 & $1855^{\circ}$ titre & VIII & 1811 \\
\hline $44^{\prime \prime}$ litre & v & 1807 & $186^{\circ}$ lille & XVIII & 1817 \\
\hline $45^{\circ \prime}$ lille & & 1828 & $193^{\circ}$ titre & VIII & 1811 \\
\hline $51^{\prime \prime}$ litro & $X \mathrm{LIN}$ & 1827 & $204^{\prime \prime}$ titre & IX & 1812 \\
\hline $53^{\prime \prime}$ tille & $X V I$ & 1815 & $2055^{\circ}$ litre & $X I$ & 1812 \\
\hline $57^{\text {c゙ litre }}$ & IV & 1807 & $208^{\circ}$ litre & $\mathrm{X}_{1}$ & 1812 \\
\hline $5^{8 \prime}$ litro & $v$ & 1807 & $20 g^{10}$ titre & $\mathrm{XIV}$ & 1814 \\
\hline $59^{\circ}$ litlc & VII & 1810 & $2100^{\prime \prime}$ litre & II & 1806 \\
\hline 60$)^{\circ}$ & vil & 1810 & $211^{\circ}$ titre & $x x$ & 1818 \\
\hline $62^{\prime \prime}$ litre & $X V I$ & 1815 & $216^{\prime \prime}$ titre & 1 & 1839 \\
\hline $63^{\circ}$ litre & $x_{1}$ & 1812 & $218^{\circ}$ litre & $x \vee 1$ & 1815 \\
\hline $65^{\circ}$ line & $\mathrm{x}$ & 1812 & 219 "ritre & XIV & 1814 \\
\hline $66^{\circ}$ titro & XII & 1812 & $222^{\prime \prime}$ titre & XIIIV & 1827 \\
\hline $68^{\circ}$ ritro & XVIII & 1817 & $223^{\prime \prime}$ inre & XLIV & 1827 \\
\hline $6 g^{\prime \prime}$ litre: & XIX & 1817 & $226^{\prime \prime}$ ritre: & XVI & 1815 \\
\hline $72^{\prime \prime}$ illoc & $\mathrm{xx}$ & 1818 & $238^{80}$ litre & IX & 1812 \\
\hline $73^{\circ \prime}$ line & $\mathrm{XI}$ & 1812 & $240^{\circ "}$ litre & VIII & 1811 \\
\hline $75^{\circ}$ tille & I & 1806 & $211^{\prime \prime}$ titre & $\mathrm{XII}$ & 1812 \\
\hline $76^{\prime \prime}$ litre & $x$ & 1812 & $244^{\prime \prime}$ litre & $x \vee 1$ & 1815 \\
\hline $78^{\prime \prime}$ line & $X V I$ & 1815 & $246)^{\prime \prime}$ titre & $X V I$ & 1815 \\
\hline $79^{\prime \prime}$ line & 1 & 1806 & $247^{\circ}$ litre & $\mathrm{x}$ & 1812 \\
\hline $80^{\prime \prime}$ lit! & $\mathrm{x}$ & 1812 & $24^{8}$ titue & $\mathrm{x} 111$ & 1812 \\
\hline $83^{\circ}$ titre & XVIII & 1817 & 250 titre & $\mathrm{X} 111$ & 1812 \\
\hline 85 "ille & $x \times 111$ & 1819 & $2.5 .3^{\circ}$ line & $x$ & 1812 \\
\hline $88^{\circ}$ litre & IV & 1807 & 255 titre & III & 1806 \\
\hline $89^{\prime \prime}$ เाн & III & 1806 & $257^{\circ}$ titre & VI & 1808 \\
\hline $92^{\circ}$ IIIIC & $x+11$ & 1812 & $25^{8^{\circ}}$ itite & IX & 1812 \\
\hline $93^{\circ}$ ॥ा & VII & 1810 & 260 litre & XIX & 1817 \\
\hline $94^{\circ}$ litre & $x \times x v$ & 1822 & 263 itre & $\mathrm{XII}$ & 1812 \\
\hline $95^{\circ}$ tilre & XII & 1812 & 267 titre & IV & 1807 \\
\hline $97^{\circ}$ litre & $\mathrm{X}_{1}$ & 1812 & $268:$ litre & III & 1806 \\
\hline $98^{\prime \prime}$ lille & $x$ & 1812 & $26 g^{\prime \prime}$ titre & I & 1806 \\
\hline $103^{\circ}$ tine & VI & 1808 & $270^{16}$ litre & II & 1806 \\
\hline $104^{\circ}$ intre & $x \times x+1$ & 1825 & 272 litre & $\mathrm{XIV}$ & 1814 \\
\hline $105^{\prime \prime}$ titre & $\mathrm{xx}$ & 1818 & $273^{\circ}$ tille & XIV & 1814 \\
\hline $106^{\prime \prime}$ titre & XVIII & 1817 & $275^{\prime \prime}$ tille & VI & 1808 \\
\hline $107^{\prime \prime} \operatorname{tinc}$ & $\mathrm{x} I \mathrm{X}$ & 1817 & $27^{8}$ titre & $X L I V$ & 1827 \\
\hline $111^{\circ}$ litre & & 1825 & $281^{\circ}$ ritre & v & 1807 \\
\hline $113^{\circ}$ litre & $v$ & $180 \%$ & $28-5$ lice & IV & 1807 \\
\hline $121^{\circ}$ litro & $x x$ & 18,8 & $286^{\circ}$ litre & $v^{\prime} I$ & 1808 \\
\hline $122^{\circ}$ litro & $\mathrm{XII}$ & 1812 & 28()$^{10}$ title & L. & 1839 \\
\hline $126^{\prime \prime}$ litre & I & 1806 & 2()$^{2}$ titre & XIII & 1812 \\
\hline $127^{\prime \prime}$ litlo & 1 & 1806 & $293^{10}$ titre & VII & 1810 \\
\hline $129^{\prime \prime}$ litre & $x x$ & 1818 & $295)^{10}$ titre & & 1828 \\
\hline $132^{\circ}$ litre & XVIII & 1817 & $206^{10} \operatorname{titrc}$ & XI & 1812 \\
\hline $133^{\prime \prime}$ titre & XIV & 1814 & $208^{\prime \prime}$ litre & $\mathrm{x} 111$ & 1812 \\
\hline 34 1itle & $x \mid x$ & 1817 & $2(9) 9^{\circ}$ ititre & VIII & 1811 \\
\hline $13^{8}$ lill & VII & 1810 & 3000 litre & $\mathrm{IX}$ & 1812 \\
\hline $139^{\circ} \operatorname{lit} 0$ & $v$ & 1807 & & & \\
\hline
\end{tabular}


Reference works which marked their day and were improvements orer what previously existed are normally taken for granted, eventually forgotten. The in part posthumous work of X.X. Willemin in selecting and publishing the 'National Antiquities' of France, is one of the earlicr and more important histories of the arts on a comparative method, treating works from the sixth (o) seventeenth centuries as documents of the historical evolution of societs. The are works discussed were non only in the leading Parisian and provincial museums and libraries, but from outstanding private collections as well, so the Momuments fransais inédits is the result of a complex series of relations between scholars, editors, collectors and dealers in the transitional period between Revolution and Restoration. Their identification and the characterization of their activities will provicle scholars with a more secure foundation for further research in the carly Romantic period which is as yet poorly documented. 